\title{
Lévy-Student Distributions for Halos in Accelerator Beams
}

\author{
Nicola Cufaro Petron* \\ Dipartimento di Matematica dell'Università di Bari, \\ and INFN Sezione di Bari, \\ via E. Orabona 4, 70125 Bari, Italy
}

\author{
Salvatore De Martino 0 Silvio De Siena ${ }^{7}$ and Fabrizio Illuminat $\$$ \\ Dipartimento di Fisica dell'Università di Salerno, \\ INFM Unità di Salerno and INFN Sezione di Napoli - Gruppo collegato di Salerno, \\ Via S. Allende, I-84081 Baronissi (SA), Italy
}

(Dated: October 28, 2005)

\begin{abstract}
We describe the transverse beam distribution in particle accelerators within the controlled, stochastic dynamical scheme of the Stochastic Mechanics (SM) which produces time reversal invariant diffusion processes. This leads to a linearized theory summarized in a Shchrödinger-like $(\mathrm{S}-\ell)$ equation. The space charge effects have been introduced in a recent paper [1] by coupling this $\mathrm{S}-\ell$ equation with the Maxwell equations. We analyze the space charge effects to understand how the dynamics produces the actual beam distributions, and in particular we show how the stationary, self-consistent solutions are related to the (external, and space-charge) potentials both when we suppose that the external field is harmonic (constant focusing), and when we a priori prescribe the shape of the stationary solution. We then proceed to discuss a few new ideas 2] by introducing the generalized Student distributions, namely non-Gaussian, Lévy infinitely divisible (but not stable) distributions. We will discuss this idea from two different standpoints: (a) first by supposing that the stationary distribution of our (Wiener powered) SM model is a Student distribution; (b) by supposing that our model is based on a (non-Gaussian) Lévy process whose increments are Student distributed. We show that in the case (a) the longer tails of the power decay of the Student laws, and in the case (b) the discontinuities of the Lévy-Student process can well account for the rare escape of particles from the beam core, and hence for the formation of a halo in intense beams.
\end{abstract}

PACS numbers: 02.50.Ey, 05.40.Fb, 29.27.Bd, 41.75.Lx

\section{INTRODUCTION}

In high intensity beams of charged particles, proposed in recent years for a wide variety of accelerator-related applications, it is very important to keep at low level the beam loss to the wall of the beam pipe, since even small fractional losses in a high-current machine can cause exceedingly high levels of radioactivation. It is now widely believed that one of the relevant mechanisms for these losses is the formation of a low intensity beam halo more or less far from the core. These halos have been observed [3] or studied in experiments [4], and have also been subjected to an extensive simulation analysis [5]. For the next generation of high intensity machines it is however still necessary to obtain a more quantitative understanding not only of the physics of the halo, but also of the beam transverse distribution in general [ $[$ ]. In fact "because there is not a consensus about its definition, halo remains an imprecise term" [] so that several proposals have been put forward for its description.

The charged particle beams are usually described in

\footnotetext{
*Electronic address: cufaro@ba.infn.it

${ }^{\dagger}$ Electronic address: demartino@sa.infn.it

$\ddagger$ Electronic address: desiena@sa.infn.it

$\S$ Electronic address: illuminati@sa.infn.it
}

terms of classical, deterministic dynamical systems. The standard model is that of a collisionless plasma where the corresponding dynamics is embodied in a suitable phase space (see for example [8]). In this framework the beam is studied by means of the particle-in-core (pic) model and the simulations show that the instabilities due to a parametric resonance can allow the particles to escape from the core with consequent halo formation [5, [6, 7]. The present paper takes a different approach: it follows the idea that the particle trajectories are samples of a stochastic process, rather than usual deterministic (differentiable) trajectories. In the usual dynamical models there is a particle probability distribution obeying the Vlasov equation, and its evolution is Liouvillian in the sense that the origin of the randomness is just in the initial conditions: along the time evolution, which is supposed to be deterministic, there is no new source of uncertainty. It is the non linear character of the equations which produces the possible unpredictable character of the trajectories. On the other hand in our model the trajectories are replaced by stochastic processes since the time evolution is supposed to be randomly perturbed even after the initial time. It is open to discussion which one of these two description is more realistic; in particular we should ask if the mutual interactions among the beam particles look like random collisions, or rather like continuous deterministic interactions. In the opinion of the authors, however, a plasma (with collisions) described in 
terms of controlled stochastic processes is a good candidate to explain the rare escape of particles from a quasistable beam core by statistically taking into account the random inter-particle interactions that can not be described in detail. Of course the idea of a stochastic approach is hardly new $[8,[9]$, but there are several different ways to implement it.

First of all let us remark that the system we want to describe is endowed with some measure of invariance under time reversal, since the external fields act to keep it in a quasi-stationary non diffusive state despite the repulsive electro-magnetic (e.m.) interactions among the constituent particles. However, a widespread misconception notwithstanding, a theory of stochastic processes not always describe irreversible systems: the addition of a dynamics to a stochastic kinematics can in fact ascribe a measure of time reversal invariance also to a stochastic system [10]. The standard way to build a stochastic dynamical system is to modify the phase space dynamics by adding a Wiener noise $\mathbf{B}(t)$ to the momentum equation only, so that the usual relations between position and velocity is preserved:

$$
\begin{aligned}
m d \mathbf{Q}(t) & =\mathbf{P}(t) d t, \\
d \mathbf{P}(t) & =\mathbf{F}(t) d t+\beta d \mathbf{B}(t) .
\end{aligned}
$$

In this way we get a derivable, but not Markovian position process $\mathbf{Q}(t)$. The standard example of this approach is that of a Brownian motion in a force field described by an Ornstein-Uhlenbeck system of stochastic differential equations (SDE) 11]. Alternatively we can add a Wiener noise $\mathbf{W}(t)$ with diffusion coefficient $D$ to the position equation:

$$
d \mathbf{Q}(t)=\mathbf{v}_{(+)}(\mathbf{Q}(t), t) d t+\sqrt{D} d \mathbf{W}(t) .
$$

and get a Markovian, but not derivable $\mathbf{Q}(t)$. In this way the stochastic system is also reduced to a single SDE since we are obliged to drop the second (momentum) equation: in fact now $\mathbf{Q}(t)$ is no more derivable. The standard example of this reduction is the Smoluchowski approximation of the Ornstein-Uhlenbeck process in the overdamped case [11]. As a consequence we will work only in a configuration, and not in a phase space; but this does not prevent us from introducing a dynamics as we will show in the Section $1 \mathrm{~A}$ - either by generalizing the Newton equations 11, 12, or by means of a stochastic variational principle [13]. Remark that in this scheme the forward velocity $\mathbf{v}_{(+)}(\mathbf{r}, t)$ can no more be an a priori given field: rather it now plays the role of a new dynamical variable of our system. This second scheme, the Stochastic mechanics (SM), is universally known for its original application to the problem of building a classical stochastic model for Quantum Mechanics (QM), but in fact it is a very general model which is suitable for a large number of stochastic dynamical systems [10, 14]. We will also see in the Section $\llbracket A$ that from the stochastic variational principles two coupled equations are derived which are equivalent to a Schrödinger-like (S- $\ell$ ) differential equation: in this sense we will speak of quantum-like
$(\mathrm{Q}-\ell)$ systems, in analogy with other recent researches on this subject [15, 16]. In fact the SM can be used to describe every stochastic dynamical system satisfying fairly general conditions: it is known since longtime [17], for example, that for any given diffusion there is a correspondence between diffusion processes and solutions of $S-\ell$ equations where the Hamiltonians come in general from suitable vector potentials. Under some regularity conditions this correspondence is seen to be one-to-one. The usual Schrödinger equation, and hence QM, is recovered when the diffusion coefficient coincides with $\hbar / 2 m$, namely is connected to the Planck constant. However we are interested here not in a stochastic model of QM, but in the description of particle beams.

In the present paper we intend to widen the scope of our SM model by introducing the idea that an important role for the beam dynamics can be played by non-Gaussian Lévy distributions. In fact these distributions enjoyed a widespread popularity in the recent years because of their multifaceted possible applications to a large set of problems from the statistical mechanics to the mathematical finance (see for example [10, 18] and references quoted therein). In particular the so called stable laws (see Section $\mathrm{A}$ ) are used in a large number of instances, as for example in the definition of the so-called Lévy flights. Our research is instead focused on a family of non-Gaussian Lévy laws which are infinitely divisible but not stable: the generalize Student laws. As will be discussed later this will allow us to overcome - without resorting to the trick of the truncated laws - the problems raised by the fact that the stable non-Gaussian laws always have divergent variances: a feature which is not realistic to ascribe to most real systems. It is possible to show indeed that by suitably choosing the parameters of the Student laws we can have distributions with finite variance, and approximating the Gaussian law as well as we want. On the other hand the infinitely divisible character of these laws is all that is required to build a stationary, stochastically continuous Markov process with independent increments, namely the Lévy process that we propose to use to represent the evolution of our particle beam.

Of course it is not always mathematically easy to deal with the infinitely divisible processes, but we will show that at least in two respects they will help us to have some further insight in the beam dynamics. First of all we use the Student distributions in the framework of the traditional SM where the randomness of process is supplied by a Gaussian Wiener noise: here we examine the features of the self-consistent potentials which can produce a Student distribution as stationary transverse distribution of a particle beam. In this instance the focus of our research is on the increase of the probability of finding the particles at a great distance from the beam core. Then we pass to the definition of a true Lévy-Student process, and we show with a few simulations that these processes can help to explain how a particle can be expelled from the bunch because of some kind of hard collision. In fact the 
trajectories of our Lévy-Student process show the typical jumps of the non-Gaussian Lévy processes: a feature that we propose to use as a model for the halo formation. It is worth remarking that, albeit the more recent empirical data about halos 19] are still not accurate enough to distinguish between the suggested distributions and the usual Gaussian ones, our conjecture on the role of Student laws in the transverse beam dynamics has recently found a first confirmation [20] in numerical simulations showing how these laws are well suited to describe the statistics of the random features of the particle paths.

In a few previous papers [21] we connected the (transverse) r.m.s. emittance to the characteristic microscopic scale and to the total number of the particles in a bunch, and implemented a few techniques of active control for the dynamics of the beam. In this paper we first of all review the theoretical basis [1, 21] of the proposed model: in the Section [I we define our SM model with emphasis added on the potentials which control the beam dynamics and on the possible non stationary solutions of this model 22]. In the Section [II] we review our analysis of the self-consistent, space charge effects due to the e.m. interaction among the particles, adding a few new results and comments. In the Section [IV we then discuss the idea [2] that the laws ruling the transverse distribution of particle beams are non-Gaussian, infinitely divisible, Lévy laws as the generalized Student laws. In particular we analyze the behavior of our usual SM model under the hypothesis that the stationary transverse distribution is a Student law. Finally in the Section $\nabla$ we study the possibility of extending our SM model to Lévy processes whose increments are distributed according to the Student law. We think in particular that the presence of isolated jumps in the trajectories can help to build a realistic model for the possible formation of halos in the particle beams. We end the paper with a few conclusive remarks.

\section{STOCHASTIC BEAM DYNAMICS}

\section{A. Stochastic mechanics}

First of all we introduce the stochastic process performed by a representative particle that oscillates around the closed ideal orbit in a particle accelerator. We consider the 3-dimensional (3-DIM) diffusion process $\mathbf{Q}(t)$, taking the values $\mathbf{r}$, which describes the position of the representative particle and whose probability density is proportional to the particle density of the bunch. As stated in the Section प the evolution of this process is ruled by the Itô stochastic differential equation (SDE)

$$
d \mathbf{Q}(t)=\mathbf{v}_{(+)}(\mathbf{Q}(t), t) d t+\sqrt{D} d \mathbf{W}(t),
$$

where $\mathbf{v}_{(+)}(\mathbf{r}, t)$ is the forward velocity, and $d \mathbf{W}(t) \equiv$ $\mathbf{W}(t+d t)-\mathbf{W}(t)$ is the increment process of a standard Wiener noise $\mathbf{W}(t)$; as it is well known this increment process is gaussian with law $\mathcal{N}(0, \mathbb{I} d t)$, where $\mathbb{I}$ is the $3 \times 3$ identity matrix. Finally the diffusion coefficient $D$ is supposed to be constant: the quantity $\alpha=2 m D$, which has the dimensions of an action, will be later connected to the characteristic transverse emittance of the beam. The equation (11) defines the random kinematics performed by the particle, and replaces the usual deterministic kinematics

$$
d \mathbf{q}(t)=\mathbf{v}(\mathbf{q}(t), t) d t
$$

where $\mathbf{q}(t)$ is just the trajectory in the 3-DIM space.

To counteract the dissipation due to this stochastic kinematics, a dynamics must be independently added. In SM we do not have a phase space: our description is entirely in a 3-DIM configuration space. This means in particular that the dynamics is not introduced in a Hamiltonian way, but by means of a suitable stochastic least action principle 13. obtained as a generalization of the variational principle of classical mechanics. In the following we will briefly review the main results, referring for details to the references [10, 11, 13]. Given the SDE (1), we consider the probability density function (pdf) $\rho(\mathbf{r}, t)$ associated to the diffusion $\mathbf{Q}(t)$ so that, besides the forward velocity $\mathbf{v}_{(+)}(\mathbf{r}, t)$, we can now define a backward velocity

$$
\mathbf{v}_{(-)}(\mathbf{r}, t)=\mathbf{v}_{(+)}(\mathbf{r}, t)-2 D \frac{\nabla \rho(\mathbf{r}, t)}{\rho(\mathbf{r}, t)} .
$$

We can then introduce also the current and the osmotic velocity fields, defined as:

$$
\mathbf{v}=\frac{\mathbf{v}_{(+)}+\mathbf{v}_{(-)}}{2} ; \quad \mathbf{u}=\frac{\mathbf{v}_{(+)}-\mathbf{v}_{(-)}}{2}=D \frac{\nabla \rho}{\rho} .
$$

Here $\mathbf{v}$ represents the velocity field of the density, while $\mathbf{u}$ is of intrinsic stochastic nature and is a measure of the non differentiability of the stochastic trajectories.

A first consequence of the stochastic generalization of the least action principle [11, 13] is that the current velocity takes the following irrotational form:

$$
m \mathbf{v}(\mathbf{r}, t)=\nabla S(\mathbf{r}, t)
$$

while the Lagrange equations of motion for the density $\rho$ and for the current velocity $\mathbf{v}$ are the continuity equation associated to every stochastic process

$$
\partial_{t} \rho=-\nabla \cdot(\rho \mathbf{v})
$$

and a dynamical equation

$$
\partial_{t} S+\frac{m}{2} \mathbf{v}^{2}-2 m D^{2} \frac{\nabla^{2} \sqrt{\rho}}{\sqrt{\rho}}+V(\mathbf{r}, t)=0,
$$

which characterizes our particular class of time-reversal invariant diffusions (Nelson processes). The last equation has the same form of the Hamilton-JacobiMadelung (HJM) equation, originally introduced in the 
hydrodynamic description of quantum mechanics by Madelung [23]. Since (5) holds, the two equations (6) and (7) can be put in the following form

$$
\begin{aligned}
\partial_{t} \rho & =-\frac{1}{m} \nabla \cdot(\rho \nabla S) \\
\partial_{t} S & =-\frac{1}{2 m} \nabla S^{2}+2 m D^{2} \frac{\nabla^{2} \sqrt{\rho}}{\sqrt{\rho}}-V(\mathbf{r}, t)
\end{aligned}
$$

which now constitutes a coupled, non linear system of partial differential equations for the pair $(\rho, S)$ which completely determines the state of our beam. On the other hand, because of (5), this state is equivalently given by the $\operatorname{pair}(\rho, \mathbf{v})$.

It can also be shown by simple substitution from (4) that (6) is equivalent to the standard Fokker-Planck (FP) equation

$$
\partial_{t} \rho=-\nabla \cdot\left[\mathbf{v}_{(+)} \rho\right]+D \nabla^{2} \rho
$$

formally associated to the Itô equation (1). In fact also the HJM equation (7) can be cast in a form based on $\mathbf{v}_{(+)}$rather than on $\mathbf{v}$, namely

$$
\begin{aligned}
\partial_{t} S=-\frac{m}{2} \mathbf{v}_{(+)}^{2}+ & m D \mathbf{v}_{(+)} \nabla \ln f \\
+ & m D^{2} \nabla^{2} \ln f-V
\end{aligned}
$$

where $f$ is a dimensionless density defined by

$$
\rho(\mathbf{r}, t)=C f(\mathbf{r}, t)
$$

where $C$ is a dimensional constant. On the other hand, from (3) and (4), we know that also the forward velocity $\mathbf{v}_{(+)}$is irrotational:

$$
\mathbf{v}_{(+)}(\mathbf{r}, t)=\nabla W(\mathbf{r}, t),
$$

and that by taking (5) into account the functions $W$ and $S$ are connected by the relation

$$
S(\mathbf{r}, t)=m W(\mathbf{r}, t)-m D \ln f(\mathbf{r}, t)-\theta(t)
$$

where $\theta$ is an arbitrary function of $t$ only.

The time-reversal invariance is now made possible [12] by the fact that the forward drift velocity $\mathbf{v}_{(+)}(\mathbf{r}, t)$ is no more an a priori given field, as is usual for the diffusion processes of the Langevin type; instead it is dynamically determined at any instant of time, starting by an initial condition, through the HJM evolution equation (7). It is finally important to remark that, introducing the representation 23$]$

$$
\Psi(\mathbf{r}, t)=\sqrt{\rho(\mathbf{r}, t)} \mathrm{e}^{i S(\mathbf{r}, t) / \alpha},
$$

(with $\alpha=2 m D$ ) the coupled equations (8) and (9) are made equivalent to a single linear equation of the form of the Schrödinger equation, with the Planck action constant replaced by $\alpha$ :

$$
i \alpha \partial_{t} \Psi=-\frac{\alpha^{2}}{2 m} \nabla^{2} \psi+V \Psi .
$$

We will refer to it as a Schrödinger-like $(\mathrm{S}-\ell)$ equation: clearly (16) has not the same meaning as the usual Schrödinger equation; this would be true only if $\alpha=\hbar$, while in general $\alpha$ is not an universal constant, and it is rather a quantity characteristic of the system under consideration (in our case the particle beam). In fact $\alpha$ turns out to be of the order of magnitude of the beam emittance, a quantity which - in formal analogy with $\hbar$ - has the dimensions of an action and gives a measure of the position/momentum uncertainty product for the system. Thus the SM model of our beam, as incorporated in the phenomenological Schrödinger equation (16), while keeping a few features reminiscent of the QM, is in fact a deeply different theory.

\section{B. Controlled distributions}

We have introduced the equations that in the SM model are supposed to describe the dynamical behavior of the beam: we now briefly sum up a general procedure, already exploited in previous papers 21, 24], to control the dynamics of our systems. Let us suppose that the pdf $\rho(\mathbf{r}, t)$ be given all along its time evolution: think in particular either to a stationary state, or to an engineered evolution from some initial pdf toward a final state with suitable characteristics. We know that the FP equation (10) must be satisfied, for the given $\rho$, by some forward velocity field $\mathbf{v}_{(+)}(\mathbf{r}, t)$. Since also the equation (13) must hold, we are first of all required to find an irrotational $\mathbf{v}_{(+)}$which satisfies the FP equation (10) for the given $\rho$. We then take into account also the dynamical equation (11): since $\rho$ and $\mathbf{v}_{(+)}$(and hence $f$ and $W$ ) are now fixed and satisfy (10), the equation (11) plays the role of a constraint defining a controlling potential $V$ when we also take into account the equation (14). We list here the potentials associated to the three particular cases analyzed in the previous papers.

In the 1-DIM case with given dimensionless pdf $f(x, t)$ and $a<x<b$ ( $a$ and $b$ can be infinite) we easily get

$$
\begin{aligned}
v_{(+)}(x, t)= & D \frac{\partial_{x} \rho(x, t)}{\rho(x, t)}-\frac{1}{\rho(x, t)} \int_{a}^{x} \partial_{t} \rho\left(x^{\prime}, t\right) d x^{\prime} \\
V(x, t)= & m D^{2} \partial_{x}^{2} \ln f+m D\left(\partial_{t} \ln f+v_{(+)} \partial_{x} \ln f\right) \\
& -\frac{m}{2} v_{(+)}^{2}-m \int_{a}^{x} \partial_{t} v_{(+)}\left(x^{\prime}, t\right) d x^{\prime}+\dot{\theta}
\end{aligned}
$$

For a 3-DIM system with cylindrical symmetry around the $z$-axis (the beam axis), if we denote with $(r, \varphi, z)$ the cylindrical coordinates, and if we suppose that $\rho(r, t)$ depends only on $r$ and $t$, and that $\mathbf{v}_{(+)}=v_{(+)}(r, t) \hat{\mathbf{r}}$ is radially directed with modulus depending only on $r$ and 
$t$, we have

$$
\begin{aligned}
v_{(+)}(r, t)= & D \frac{\partial_{r} \rho(r, t)}{\rho(r, t)}-\frac{1}{r \rho(r, t)} \int_{0}^{r} \partial_{t} \rho\left(r^{\prime}, t\right) r^{\prime} d r^{\prime} \\
V(r, t)= & \frac{m D^{2}}{r} \partial_{r}\left(r \partial_{r} \ln f\right)+m D\left(\partial_{t} \ln f+v_{(+)} \partial_{r} \ln f\right) \\
& -\frac{m}{2} v_{(+)}^{2}-m \int_{0}^{r} \partial_{t} v_{(+)}\left(r^{\prime}, t\right) d r^{\prime}+\dot{\theta}
\end{aligned}
$$

Finally in the 3-DIM stationary case the pdf $\rho(\mathbf{r})$ is independent from $t$. This greatly simplifies our formulas and, by requiring that $\dot{\theta}(t)=E$ be constant, namely that $\theta(t)=E t$, we get

$$
\begin{aligned}
\mathbf{v}_{(+)}(\mathbf{r}) & =D \frac{\nabla \rho(\mathbf{r})}{\rho(\mathbf{r})} \\
V(\mathbf{r}) & =E+2 m D^{2} \frac{\nabla^{2} \sqrt{\rho}}{\sqrt{\rho}} .
\end{aligned}
$$

Of course in this context the constant $E$ will be chosen by fixing the zero of the potential energy. Let us remark finally that in this stationary case the phenomenological wave function (15) takes the form

$$
\Psi(\mathbf{r}, t)=\sqrt{\rho} e^{-i E t / \alpha}
$$

typical of the stationary states.

\section{Non stationary distributions}

In the following we will be mainly concerned with stationary distributions, but in a few previous paper we treated also non stationary problems. For instance, if we consider the stationary, ground state pdf (without nodes) $\rho_{0}(\mathbf{r})$ of a suitable potential, and if we calculate $\mathbf{v}_{(+)}(\mathbf{r})$ and write down the corresponding FP equation, it is possible to show (see the general proof in a few previous papers $24,25,26])$ that, $\rho_{0}(\mathbf{r})$ will play the role of an attractor for every other distribution (non extremal with respect to a stochastic minimal action principle). If the accelerator beam is ruled by such an equation, this would imply that the halo can not simply be wiped out by scraping away the particles that come out of the bunch core: in fact they simply will keep going out in the halo until the equilibrium is reached again since the distribution $\rho_{0}(\mathbf{r})$ is a stable attractor.

In a recent paper 22] we gave an estimate of the time required for the relaxation of non extremal pdf's toward the equilibrium distribution. This is an interesting test for our model since this relaxation time is fixed once the form of the forward velocity field is given; this is in turn fixed when the form of the halo distribution is given as in the reference [1], and one could check if the estimate is in agreement with possible observed times. In particular we estimated that in typical conditions all the non-stationary solutions of this FP equation will be attracted toward $\rho_{0}$ with a relaxation time of the order of $\tau \approx 2 m \sigma^{2} / \alpha \approx 10^{-8} \div 10^{-7}$ sec.
A different non stationary problem also discussed in previous papers [21, 22] consists in the analysis of some particular time evolution of the process with the aim of finding the dynamics that control it. For instance we studied the possible evolutions which start from a pdf with halo and evolve toward a halo-free pdf: this would allow us to find the dynamics that we are requested to apply in order to achieve this result. If for simplicity the overall process is supposed to be an Ornstein-Uhlenbeck process, the transition pdf would be completely known and all the result can be exactly calculated through the Chapman-Kolmogorov equation by supposing suitable shapes for the initial and final distributions. Then a direct application of (18) allows us to calculate the control potential corresponding to this evolution. For the sake of brevity we do not give the analytical form of this potential and refer to the quoted papers for further details.

\section{SELF-CONSISTENT EQUATIONS}

\section{A. Space charge interaction}

In QM a system of $N$ particles is described by a wave function in a $3 N-D I M$ configuration space. On the other hand in our SM scheme a normalized $|\Psi(\mathbf{r}, t)|^{2}$, function of only three space coordinates $\mathbf{r}=\{x, y, z\}$, plays the role of the pdf of a Nelson process. In a first approximation we will consider this $N$-particle system as a pure ensemble: as a consequence we will not introduce a $3 N-$ DIM configuration space, since $N|\Psi(\mathbf{r}, t)|^{2} d^{3} \mathbf{r}$ in the 3-DIM space will play the role of the number of particles in a small neighborhood of $\mathbf{r}$. However, since our system of $N$ charged particles is not a pure ensemble due to their mutual e.m. interaction, in a further mean field approximation we will take into account the so called space charge effects: more precisely we will couple our S$\ell$ equation with the Maxwell equations describing both the external and the space charge e.m. fields, and we will get in the end a non linear system of coupled differential equations.

In our model a single, charged particle embedded in a beam and feeling both an external, and a space charge potential is first of all described by a $\mathrm{S}-\ell$ equation

$$
i \alpha \partial_{t} \Psi(\mathbf{r}, t)=\widehat{H} \Psi(\mathbf{r}, t),
$$

where $\Psi(\mathbf{r}, t)$ is our wave function, $\alpha$ a coefficient with the dimensions of an action which is a constant depending on the beam characteristics, and $\widehat{H}$ is a suitable Hamiltonian operator. If $\Psi$ is properly normalized and if $N$ is the number of particles with individual charge $q_{0}$, the space charge density and the electrical current density are

$$
\begin{aligned}
\rho_{s c}(\mathbf{r}, t) & =N q_{0}|\Psi(\mathbf{r}, t)|^{2}, \\
\mathbf{j}_{s c}(\mathbf{r}, t) & =N q_{0} \frac{\alpha}{m} \Im\left\{\Psi^{*}(\mathbf{r}, t) \nabla \Psi(\mathbf{r}, t)\right\} .
\end{aligned}
$$

Hence our particles in the beam will feel both an electrical and a magnetic interaction and we will be obliged to 
couple the $S-\ell$ equation with the equations of the vector and scalar potentials associated to this electro-magnetic field.

The e.m. potentials $\left(\mathbf{A}_{s c}, \Phi_{s c}\right)$ of the space charge fields obeying the gauge condition

$$
\nabla \cdot \mathbf{A}_{s c}(\mathbf{r}, t)+\frac{1}{c^{2}} \partial_{t} \Phi_{s c}(\mathbf{r}, t)=0,
$$

must satisfy the wave equations

$$
\begin{aligned}
\nabla^{2} \mathbf{A}_{s c}(\mathbf{r}, t)-\frac{1}{c^{2}} \partial_{t}^{2} \mathbf{A}_{s c}(\mathbf{r}, t) & =-\mu_{0} \mathbf{j}_{s c}(\mathbf{r}, t) \\
\nabla^{2} \Phi_{s c}(\mathbf{r}, t)-\frac{1}{c^{2}} \partial_{t}^{2} \Phi_{s c}(\mathbf{r}, t) & =-\frac{\rho_{s c}(\mathbf{r}, t)}{\epsilon_{0}}
\end{aligned}
$$

On the other hand, for our particle in the beam the e.m. field is the superposition of the space charge potential $\left(\mathbf{A}_{s c}, \Phi_{s c}\right)$, and of the external potentials $\left(\mathbf{A}_{e}, \Phi_{e}\right)$. Hence (see for example [27], chapter XV) our $\mathrm{S}-\ell$ equation takes the form

$$
\begin{aligned}
i \alpha \partial_{t} \Psi=\frac{1}{2 m}\left[i \alpha \nabla-\frac{q_{0}}{c}\left(\mathbf{A}_{s c}\right.\right. & \left.\left.+\mathbf{A}_{e}\right)\right]^{2} \Psi \\
& +q_{0}\left(\Phi_{s c}+\Phi_{e}\right) \Psi
\end{aligned}
$$

It is apparent now that (25), (26), (27) and (28) constitute a self-consistent system of non linear differential equations for the fields $\Psi, \mathbf{A}_{s c}$ and $\Phi_{s c}$ coupled through (23) and (24).

If we then consider stationary wave functions

$$
\Psi(\mathbf{r}, t)=\psi(\mathbf{r}) e^{-i E t / \alpha}
$$

where $E$ is the energy of the particle, and take $\mathbf{A}_{e}=0$ for the external interaction, passing to the potential energies

$$
V_{e}(\mathbf{r})=q_{0} \Phi_{e}(\mathbf{r}), \quad V_{s c}(\mathbf{r})=q_{0} \Phi_{s c}(\mathbf{r}),
$$

our system is reduced to only two coupled, non linear equations for the pair $\left(\psi, V_{s c}\right)$, namely

$$
\begin{aligned}
\frac{\alpha^{2}}{2 m} \nabla^{2} \psi & =\left(V_{e}+V_{s c}-E\right) \psi, \\
\nabla^{2} V_{s c} & =-\frac{N q_{0}^{2}}{\epsilon_{0}}|\psi|^{2}
\end{aligned}
$$

\section{B. Cylindrical symmetry}

We suppose now that the longitudinal motion along the $z$-axis is both decoupled from the transverse motion in the $x, y$-plane, and free with constant momentum $p_{z}$, and velocity $b_{z}=b_{0} \gg b_{x}, b_{y}$. Moreover we suppose that the beam particles will be confined in a cylindrical packet of length $L$, so that by the imposing periodic boundary conditions we will quantize the longitudinal momentum

$$
p_{z}=\frac{2 k \pi \alpha}{L}, \quad k=0, \pm 1, \pm 2, \ldots
$$

As a consequence our wave functions will take the form

$$
\psi(\mathbf{r})=\chi(x, y) \frac{e^{i p_{z} z / \alpha}}{\sqrt{L}}
$$

and our equations (30) and (31) become

$$
\begin{aligned}
\frac{\alpha^{2}}{2 m}\left(\partial_{x}^{2}+\partial_{y}^{2}\right) \chi & =\left(V_{e}+V_{s c}-E_{T}\right) \chi \\
\left(\partial_{x}^{2}+\partial_{y}^{2}\right) V_{s c} & =-\frac{N q_{0}^{2}}{L \epsilon_{0}}|\chi|^{2}=-\frac{\mathcal{N} q_{0}^{2}}{\epsilon_{0}}|\chi|^{2}
\end{aligned}
$$

where $\mathcal{N}=N / L$ is the number of particles per unit length, and $E_{T}=E-p_{z}^{2} / 2 m$ is the energy of the transverse motion. If finally our system has a cylindrical symmetry around the $z$ axis, namely if - in the cylindrical coordinate system $\{r, \varphi, z\}\left(r^{2}=x^{2}+y^{2}\right)$ - our potentials depend only on $r$, then we can separate the variables with $\chi(x, y)=u(r) \Phi(\varphi)$, the angular eigenfunctions are

$$
\Phi_{\ell}(\varphi)=\frac{e^{i \ell \varphi}}{\sqrt{2 \pi}}, \quad \ell=0, \pm 1, \pm 2, \ldots
$$

and for $\ell=0$ the equations become

$$
\begin{aligned}
\frac{\alpha^{2}}{2 m}\left(u^{\prime \prime}+\frac{u^{\prime}}{r}\right) & =\left(V_{e}+V_{s c}-E_{T}\right) u \\
V_{s c}^{\prime \prime}+\frac{V_{s c}^{\prime}}{r} & =-\frac{\mathcal{N} q_{0}^{2}}{2 \pi \epsilon_{0}} u^{2}
\end{aligned}
$$

with the following radial normalization

$$
\int_{0}^{+\infty} r u^{2}(r) d r=1
$$

Remark that now we are reduced to a system of ordinary differential equations.

\section{Dimensionless formulation}

To eliminate the physical dimensions one introduces two quantities $\eta$ and $\lambda$ which are respectively an energy and a length. Then, by means of the dimensionless quantities

$$
\begin{array}{r}
s=\frac{r}{\lambda}, \quad \beta=\frac{E_{T}}{\eta}, \quad \xi=\frac{\mathcal{N} q_{0}^{2}}{2 \pi \epsilon_{0} \eta} \quad \text { (perveance) } \\
w(s)=\lambda u(\lambda s) \\
v(s)=\frac{V_{s c}(\lambda s)}{\eta}, \quad v_{e}(s)=\frac{V_{e}(\lambda s)}{\eta}
\end{array}
$$

the equations (36) and (37) take the form

$$
\begin{aligned}
s w^{\prime \prime}(s)+w^{\prime}(s) & =\left[v_{e}(s)+v(s)-\beta\right] s w(s) \\
s v^{\prime \prime}(s)+v^{\prime}(s) & =-\xi s w^{2}(s)
\end{aligned}
$$

The usual choice for the dimensional constants is

$$
\eta=m b_{0}^{2}, \quad \lambda=\frac{\alpha}{m b_{0} \sqrt{2}},
$$


where $b_{0}$ is the longitudinal velocity of the beam. We can now look at our equations in two different ways. First of all we can suppose that $v_{e}$ is a given external potential: in this case our aim is to solve the equations for the two unknowns $w$ (radial particle distribution) and $v$ (space charge potential energy). However in general no simple analytical solution of this problem is at present available for the usual forms of the external potential $v_{e}$ : there are not even solutions playing the same role played by the Kapchinskij-Vladimirskij (KV) distribution in the usual models. This phase space distribution - which is simple and self-consistent in the usual dynamical models - leads to an uniform transverse space distribution of the beam, and is a stationary solution of the Vlasov equation with a harmonic potential. Moreover its space charge potential calculated from the Poisson equation is still harmonic. Instead in the SM model the uniform distributions are not solutions of the stationary Schrödinger equation, and we know no simple stationary distribution connected to the harmonic potential as the KV. Even the gaussian distributions - later discussed in this paper - can not play the same role: they are solutions connected with an external harmonic potential, but their space charge potential calculated from the Poisson equation is not harmonic.

Alternatively we can assume as known a given distribution $w$, and solve our equations to find both the external and the space charge self-consistent potential energies $v_{e}$ and $v$. In this second form the problem is more simple, and analytical solutions are available. We adopted the first standpoint in a few previous papers [1] where we numerically solved the equations (38) and (39); here we will rather elaborate a few new ideas about the second one. To this end it is important to remark that the space charge potential energy

$$
v(s)=-\xi \int_{0}^{s} \frac{d y}{y} \int_{0}^{y} x w^{2}(x) d x
$$

is always a solution of the Poisson equation (39) satisfying the conditions $v\left(0^{+}\right)=v^{\prime}\left(0^{+}\right)=0$. On the other hand, by substituting (41) in the first equation (38) we readily obtain also the self-consistent form of the external potential energy

$$
\begin{aligned}
& v_{e}(s)=v_{0}(s)+\xi \int_{0}^{s} \frac{d y}{y} \int_{0}^{y} x w^{2}(x) d x, \\
& v_{0}(s)=\frac{w^{\prime \prime}(s)}{w(s)}+\frac{1}{s} \frac{w^{\prime}(s)}{w(s)}+\beta
\end{aligned}
$$

where $v_{0}(s)$ is the potential that we would have without space charge $(\xi=0)$, while the second part in the external potential (42) exactly compensate for the space charge potential.

\section{Constant focusing}

Let us suppose now that the transverse external potential $V_{e}(r)$ is a cylindrically symmetric, harmonic potential with a proper frequency $\omega$ (constant focusing), and let we also introduce the characteristic length

$$
\sigma^{2}=\frac{\alpha}{2 m \omega}
$$

which will represents a measure of the transverse dispersion of the beam. In cylindrical coordinates $\{r, \varphi\}$ in the transverse plane our potential energy is

$$
V_{e}(r)=\frac{m \omega^{2}}{2} r^{2}=\frac{\alpha^{2}}{8 m \sigma^{4}} r^{2}
$$

so that the corresponding $2-\mathrm{DIM} \mathrm{S}-\ell$ equation without space charge (zero perveance) would have as lowest eigenvalue $E_{0}=\alpha \omega$, and as ground state wave function

$$
\chi_{00}(r, \varphi)=\frac{u_{0}(r)}{\sqrt{2 \pi}}=\frac{e^{-r^{2} / 4 \sigma^{2}}}{\sigma \sqrt{2 \pi}} .
$$

Of course the self-consistent solution would be different if there is a space charge (non zero perveance). To find this solution one introduces the so called phase advance

$$
\frac{1}{\lambda_{0}}=\frac{\omega}{b_{0}}=\frac{\alpha}{2 m b_{0} \sigma^{2}}
$$

( $\lambda_{0}$ is a length) and, with the constants (40), the dimensionless form of the harmonic potential (44)

$$
\begin{aligned}
v_{e}(s) & =\frac{V_{e}(r)}{m b_{0}^{2}}=\frac{\omega^{2}}{b_{0}^{2}} r^{2}=\frac{r^{2}}{2 \lambda_{0}^{2}}=\frac{\alpha^{2}}{4 \lambda_{0}^{2} m^{2} b_{0}^{2}} s^{2}=\gamma^{2} s^{2} \\
\gamma & =\frac{\alpha}{2 \lambda_{0} m b_{0}}=\frac{\alpha \omega}{2 m b_{0}^{2}}=\frac{\sigma^{2}}{\lambda_{0}^{2}}
\end{aligned}
$$

As a consequence the equations (38) and (39) become

$$
\begin{aligned}
s w^{\prime \prime}(s)+w^{\prime}(s) & =\left[\gamma^{2} s^{2}+v(s)-\beta\right] s w(s) \\
s v^{\prime \prime}(s)+v^{\prime}(s) & =-\xi s w^{2}(s)
\end{aligned}
$$

These equations are now a coupled, non linear system which must be numerically solved since we do not know simple self-consistent solutions of the form of the KV distribution. In reference [1] we extensively analyzed these numerical solutions and we refer to this paper for details. In fact in [1] there was a small difference with respect to what has been presented here. The form of the equations to solve is the same, but the dimensionless formulation was achieved by means of two numerical constants different from (40) and drawn from the characteristics of the transverse harmonic oscillator force:

$$
\eta=\frac{\alpha^{2}}{4 m \sigma^{2}}=\frac{\alpha \omega}{2}, \quad \lambda=\sigma \sqrt{2}
$$

Then the dimensionless quantities have a different numerical value and the dimensionless equations (36) and (37) take the form

$$
\begin{aligned}
s w^{\prime \prime}(s)+w^{\prime}(s) & =\left[s^{2}+v(s)-\beta\right] s w(s) \\
s v^{\prime \prime}(s)+v^{\prime}(s) & =-\xi s w^{2}(s)
\end{aligned}
$$


since now $\gamma=1$. In any case the equations (46) and (47) can easily be turned into the equations (49) and (50), and vice versa, by means of simple transformations through the parameter $\gamma$ which turns out to be at the same time the ratio of the energy constants, and that of the squared length constants. As a consequence in the following we will always use the system (49), (50), with the advantage of simply putting $\gamma=1$ in the model.

\section{SELF-CONSISTENT POTENTIALS}

\section{A. Gaussian transverse distributions}

In the SM model it is possible to numerically integrate the Schrödinger-Poisson system (38) and (39) with a given external potential and calculate the self-consistent distributions and their space charge potentials [1]. On the other hand, if we fix a particular distribution, it is always possible to exactly calculate from these equations the external and space charge potential giving rise to that distribution. When we adopt this second alternative approach and we take as given the form of the distribution $w(s)$, the unknowns in the equations (38) and (39) are the two potential energies $v(s)$ and $v_{e}(s)$. In this case we only need to calculate the expressions (41) and (42) in terms of the given distribution $w(s)$. Of course if we take an arbitrary $w(s)$ we will not get any simple and meaningful form for the external potential $v_{e}(s)$; and on the other hand to guess the right form of $w(s)$ giving rise, for instance, exactly to a harmonic potential (44) as external potential would be tantamount to solve (42) as an integro-differential equation for a given external potential. However in a few explicit cases the results are quite simple and interesting.

Let us take as first example of a stationary wave function that of the ground state $u_{0}(r)$ of the harmonic oscillator with zero perveance given in (45). Its dimensionless representation is:

$$
w(s)=\sqrt{2} e^{-s^{2} / 2}, \quad \beta=2 \quad\left(E_{T}=\alpha \omega\right) ;
$$

which is also apparently normalized. We now want to calculate both the external and the space charge potentials that produce (51) as stationary wave function for (49) and (50). From (41), (42) and (51) we then have

$$
\begin{aligned}
& \frac{w^{\prime \prime}(s)}{w(s)}+\frac{1}{s} \frac{w^{\prime}(s)}{w(s)}+\beta=v_{0}(s)=s^{2} \\
& \int_{0}^{s} \frac{d y}{y} \int_{0}^{y} w^{2}(x) x d x=\frac{1}{2}\left[\log \left(s^{2}\right)+\mathbb{C}-\operatorname{Ei}\left(-s^{2}\right)\right]
\end{aligned}
$$

where $\mathbb{C} \approx 0.577$ is the Euler constant and

$$
\operatorname{Ei}(x)=\int_{-\infty}^{x} \frac{e^{t}}{t} d t, \quad x<0
$$

is the exponential-integral function, and hence we immediately get (see also FIG. 1)

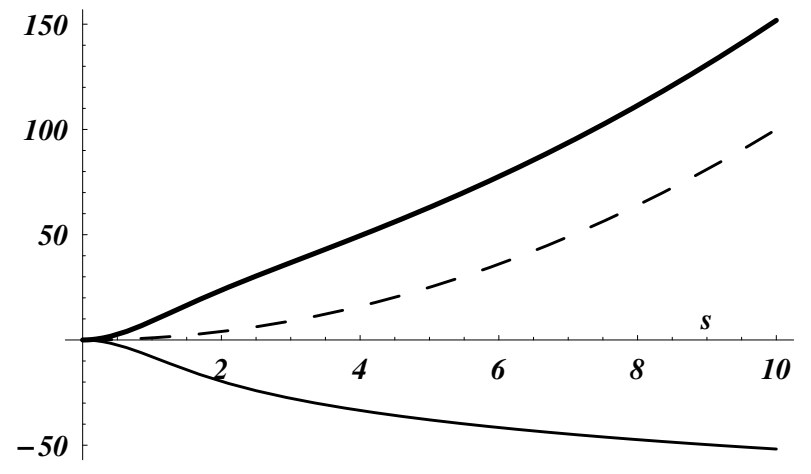

FIG. 1: The dimensionless potentials $v(s)$ (thin line), $v_{0}(s)=$ $s^{2}$ (dashed line) and $v_{e}(s)$ (thick line). They reproduce respectively equations (52), (53) and (54) for $\xi=20$ (see reference 1] for this value). When the external potential is $v_{e}(s)$ the self-consistent wave function coincides with that of a simple harmonic oscillator for zero perveance (51).

$$
\begin{aligned}
v(s) & =-\frac{\xi}{2}\left[\log \left(s^{2}\right)+\mathbb{C}-\operatorname{Ei}\left(-s^{2}\right)\right] \\
v_{0}(s) & =s^{2} \\
v_{e}(s) & =s^{2}+\frac{\xi}{2}\left[\log \left(s^{2}\right)+\mathbb{C}-\operatorname{Ei}\left(-s^{2}\right)\right]
\end{aligned}
$$

In a sense the meaning of the equations (41), (42) and (43) is rather simple: if we want to get a selfconsistent distribution which coincides with a solution of the $\mathrm{S}-\ell$ equation for a given zero perveance potential, the simplest way it is to calculate the space charge potential for this frozen distribution through the Poisson equation, and then compensate the external potential exactly for that. This is what we did in our example where the gaussian solution is the fundamental state of a harmonic oscillator: we finally got a total potential which is $v_{0}(s)=v(s)+v_{e}(s)=s^{2}$ (namely that of a simple harmonic oscillator), and an energy value which coincides with the first eigenvalue. In other words, if you want a gaussian transverse distribution you should not simply turn on a bare harmonic potential $s^{2}$ : you should rather teleologically compensate for the space charge by using the potential $v_{e}(s)$.

\section{B. Student transverse distributions}

If the halo consists in the fact that large deviations from the beam axis are possible, a new idea is to suppose that the the stationary transverse distribution is different from the gaussian distribution (51) introduced in the Section IVA To this end we will introduce in the following a family of distributions which decay with the distance from the axis only with a power law.

Let us consider the following family of univariate, twoparameters probability laws $\Sigma\left(\nu, a^{2}\right)$ characterized by the 


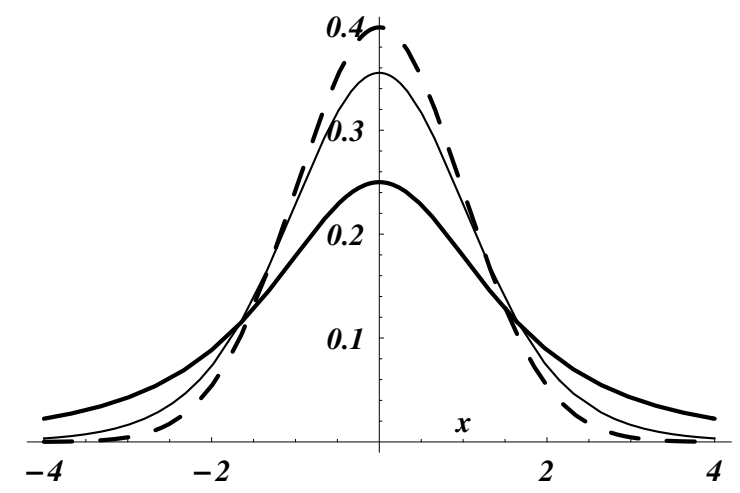

FIG. 2: The Gauss pdf $\mathcal{N}(0,1)$ (dashed line) compared with the $\Sigma(2,2)$ (thick line) and the $\Sigma(10,12)$ (thin line). The flexes of the three curves coincide. Apparently the tails of the Student laws are much longer.

following pdf's

$$
f(x)=\frac{\Gamma\left(\frac{\nu+1}{2}\right)}{\Gamma\left(\frac{1}{2}\right) \Gamma\left(\frac{\nu}{2}\right)} \frac{a^{\nu}}{\left(x^{2}+a^{2}\right)^{\frac{\nu+1}{2}}}
$$

which apparently are symmetric functions with the mode in $x=0$ and two flexes in $x= \pm a / \sqrt{\nu+2}$. All these laws are centered at the median. In particular $a$ plays just the role of a scale parameter, while $\nu$ rules the power decay of the tails: for large $x$ the tails go as $x^{-(\nu+1)}$ with $\nu+1>1$. For a comparison with a Gauss law $\mathcal{N}\left(0, \sigma^{2}\right)$ see FIG. 22 Remark that when $\nu$ grows larger and larger, the difference between the two pdf's becomes smaller and smaller. It is typical of the laws $\Sigma\left(\nu, a^{2}\right)$ that they have (finite) momenta of order $k$ only if the condition $k<\nu$ is verified; hence for $\nu \leq 2$ there is no variance, while for $\nu \leq 1$ not even the expectation is defined. On the other hand when $\nu>2$ the variance of $\Sigma\left(\nu, a^{2}\right)$ exists and is

$$
\sigma^{2}=\frac{a^{2}}{\nu-2} .
$$

It will be useful to remark that the laws $\Sigma\left(1, a^{2}\right)$ are the well-known Cauchy laws $\mathcal{C}(a)$ with pdf

$$
f(x)=\frac{1}{\pi} \frac{a}{x^{2}+a^{2}},
$$

while the laws $\Sigma(n, n)$ with $n=1,2, \ldots$ are the classical $t$-Student laws $\mathcal{S}(n)$ with pdf

$$
f(x)=\frac{\Gamma\left(\frac{n+1}{2}\right)}{\sqrt{\pi} \Gamma\left(\frac{n}{2}\right)}\left(n+x^{2}\right)^{-\frac{n+1}{2}} .
$$

We will then refer to $\Sigma\left(\nu, a^{2}\right)$ as generalized Student laws since they are just Student laws with a continuous parameter $\nu>0$ and a scale parameter $a$. For $\nu>2$ variances exist and we are then entitled to standardize our laws: indeed from (56) every $\Sigma\left(\nu,(\nu-2) \sigma^{2}\right)$ with $a^{2}=(\nu-2) \sigma^{2}$ has variance $\sigma^{2}$, and the standard (with unit variance) generalized Student laws are $\Sigma(\nu, \nu-2)$.

In order to describe the beam we will also introduce the bivariate, circularly symmetric Student laws $\Sigma_{2}\left(\nu, a^{2}\right)$ with pdf

$$
f(x, y)=\frac{\nu}{2 \pi} \frac{a^{\nu}}{\left(x^{2}+y^{2}+a^{2}\right)^{\frac{\nu+2}{2}}} .
$$

Its marginal laws are both $\Sigma\left(\nu, a^{2}\right)$ and non-correlated, albeit not independent (as in the case of the circularly symmetric gaussian bivariate laws). The total beam distribution will then be

$$
\rho(x, y, z)=\frac{1}{2 \pi L} \frac{\nu a^{\nu}}{\left(x^{2}+y^{2}+a^{2}\right)^{\frac{\nu+2}{2}}} H\left(\frac{L}{2}-|z|\right)
$$

where $H(z)$ is the Heaviside function. In the description of a beam in an accelerator it is realistic to suppose that the transverse distribution is endowed with a finite variance. Hence we will look for distributions (58) with $\nu>2$. On the other hand this will correspond to suppose that in our model the transverse Student laws should not be radically different from a Gaussian: in fact the halo is in some sense an effect which is small when compared with the total beam. From this standpoint the family of laws $\Sigma\left(\nu, a^{2}\right)$ has also the advantage that we can fine tune the parameters $\nu, a$ in order to get the right distance from the gaussian laws (this would not be possible if we adopted stable laws; see subsequent Section $\nabla A$. With this hypothesis in mind we will limit our present considerations to the case $\nu>2$ so that the transverse marginals of (58) will have a finite variance $\sigma^{2}$. Then from (56) we choose $a^{2}=(\nu-2) \sigma^{2}$ and write (58) as

$$
\rho(x, y, z)=\frac{\nu}{2 \pi L} \frac{\left[(\nu-2) \sigma^{2}\right]^{\frac{\nu}{2}}}{\left[x^{2}+y^{2}+(\nu-2) \sigma^{2}\right]^{\frac{\nu+2}{2}}} H\left(\frac{L}{2}-|z|\right)
$$

Passing to cylindrical random variables we then have

$$
\rho(r, \varphi, z)=r \frac{\nu}{2 \pi L} \frac{\left[(\nu-2) \sigma^{2}\right]^{\frac{\nu}{2}}}{\left[r^{2}+(\nu-2) \sigma^{2}\right]^{\frac{\nu+2}{2}}} H\left(\frac{L}{2}-|z|\right)
$$

namely

$$
\begin{aligned}
\rho(r, \varphi, z)=\frac{1}{\sigma \sqrt{2}} & \frac{r}{\sigma \sqrt{2}} \frac{2 \nu}{\nu-2} \\
& \times\left[1+\frac{r^{2}}{(\nu-2) \sigma^{2}}\right]^{-\frac{\nu+2}{2}} \frac{H\left(\frac{L}{2}-|z|\right)}{2 \pi L}
\end{aligned}
$$

so that finally with the shorthand notation

$$
z=\frac{s \sqrt{2}}{\sqrt{\nu-2}}
$$

the dimensionless, normalized radial distribution is

$$
w^{2}(s)=\frac{2 \nu}{\nu-2} \frac{1}{\left(1+z^{2}\right)^{\frac{\nu+2}{2}}} .
$$


Here we adopt the dimensional constants

$$
\eta=\frac{\alpha^{2}}{4 m \sigma^{2}}, \quad \lambda=\sigma \sqrt{2}
$$

where $\sigma^{2}$ is the variance of our Student laws. We can now use the relations (41), (42) and (43) in order to get the potentials which have (59) as stationary distribution: first of all the space charge potential produced by (59) has the form

$$
\begin{gathered}
v(s)=-\frac{\xi}{2}\left[\frac{2 z^{-\nu}}{\nu}{ }_{2} \mathrm{~F}_{1}\left(\frac{\nu}{2}, \frac{\nu}{2} ; \frac{\nu+2}{2} ;-\frac{1}{z^{2}}\right)\right. \\
\left.+\log z^{2}+\mathbb{C}+\psi\left(\frac{\nu}{2}\right)\right]
\end{gathered}
$$

where ${ }_{2} \mathrm{~F}_{1}(a, b ; c ; w)$ is a hypergeometric function and $\psi(w)=\Gamma^{\prime}(w) / \Gamma(w)$ is the logarithmic derivative of the Euler Gamma function (digamma function). On the other hand, by choosing $\beta=2+\frac{8}{\nu-2}$ to put the potential energies to zero in the origin, we get the control potential for zero perveance

$$
v_{0}(s)=\frac{\nu+2}{\nu-2} \frac{z^{2}\left(4 z^{2}+\nu+10\right)}{2\left(1+z^{2}\right)^{2}}
$$

and hence the external potential required to keep a transverse student distribution $\Sigma_{2}\left(\nu,(\nu-2) \sigma^{2}\right)$ with a given variance $\sigma^{2}$ is

$$
\begin{aligned}
v_{e}(s)=\frac{\nu+2}{\nu-2} & \frac{z^{2}\left(4 z^{2}+\nu+10\right)}{2\left(1+z^{2}\right)^{2}} \\
+\frac{\xi}{2} & {\left[\frac{2 z^{-\nu}}{\nu}{ }_{2} \mathrm{~F}_{1}\left(\frac{\nu}{2}, \frac{\nu}{2} ; \frac{\nu+2}{2} ;-\frac{1}{z^{2}}\right)\right.} \\
& \left.+\log z^{2}+\mathbb{C}+\psi\left(\frac{\nu}{2}\right)\right]
\end{aligned}
$$

Formulas (63), (64) and (65) give the self-consistent potentials associated with the beam distribution (59) which is transversally a Student $\Sigma_{2}\left(\nu,(\nu-2) \sigma^{2}\right)$. In the FIG. 3 we can see an example of the control potential $v_{0}(s)$ for a particular value of the parameter $\nu$, together with its limit behaviors

$$
\begin{array}{ll}
v_{0}(s) \sim \frac{(\nu+2)(\nu+10)}{(\nu-2)^{2}} s^{2}, & \left(s \rightarrow 0^{+}\right) \\
v_{0}(s) \sim \frac{(\nu+2)^{2}}{4 s^{2}}+2+\frac{8}{\nu-2}, & (s \rightarrow+\infty)
\end{array}
$$

Now this results must be compared with the similar results (52), (53) and (54) associated to a transversally gaussian distribution. We will choose the gaussian parameters in such a way that the behavior near the beam axis be similar to (66), namely (with $\beta=2 \gamma$ )

$$
w(s)=\sqrt{2 \gamma} e^{-2 \gamma s^{2} / 2}, \quad \gamma^{2}=\frac{(\nu+2)(\nu+10)}{(\nu-2)^{2}} .
$$

First of all in the FIG. 廿 we compare the space charge potential produced by both a Student and Gauss transverse distribution: remark as for the chosen parameter

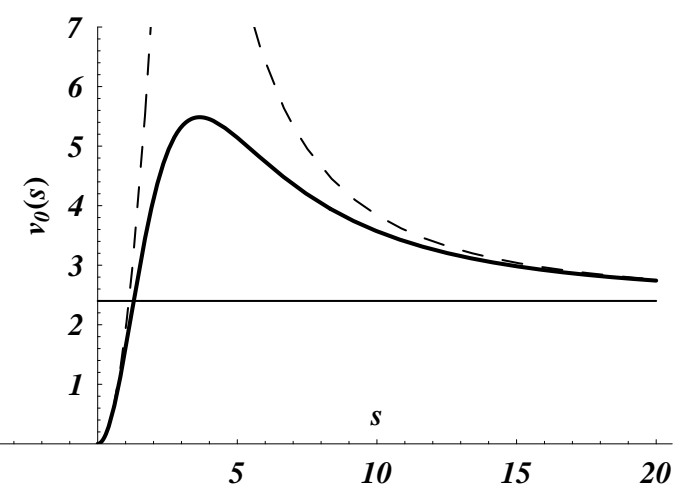

FIG. 3: The control potential $v_{0}(s)$ (64) for a Student transverse distribution $\Sigma_{2}\left(22,20 \sigma^{2}\right)$. Also displayed are the value of $\beta=2.4$ (the limit value of $v_{0}$ for large $s$, thin line) and the behaviors for small and large $s$ (66) and (67) (dashed lines).

values $(\nu=22, \xi=20)$ the two potentials look particularly similar. In fact, given the asymptotic behavior of the hypergeometric function in (63) and of the exponential integral in (52), for $s \rightarrow+\infty$ both potentials behave as $-\xi \log s$. On the other hand we immediately see from FIG. [5] that the control potentials for zero perveance $v_{0}(s)$ behave differently when we move away from the beam axis; beyond a distance of about $r \simeq 2 \sigma$ the two curves are different: while in the Gaussian case the potential diverges as $s^{2}$, in the Student case it goes to the constant value $\beta$ as quickly as $s^{-2}$. Of course this difference fades away when $\nu$ grows larger and larger; that points to the fact that the principal difference between the two cases can be confined in a region that can be made as far removed from the beam core as we want by a suitable choice of $\nu$. Finally in the FIG. 66 we compare the total external potentials needed to keep the transverse beam respectively in a Student and in a Gauss distribution. We then see that for large $s$ (far away from the beam core, while in the Gauss case the total external potential grows with $s$ as $s^{2}+\xi \log s$, in the Student case this potential only grows as $\xi \log s$. In any case, even if the potential near the beam axis is harmonic, deviations from this behavior in a region removed form the core can produce a deformation of the distribution from the gaussian to the Student.

\section{Estimating the emittance}

If $u(r)$ is a self-consistent, cylindrically symmetric solution of (36) and (37) the position probability density $\rho(r, \varphi, z)$ in cylindrical coordinates will have the form

$$
\rho(r, \varphi, z)=\frac{1}{2 \pi L} \begin{cases}u^{2}(r) & 0 \leq \varphi<2 \pi, \quad-\frac{L}{2} \leq z \leq \frac{L}{2}, \\ 0 & \text { otherwise. }\end{cases}
$$

In order to estimate the emittance we need to calculate mean values of positions $x$ and momenta $p_{x}$ along one 


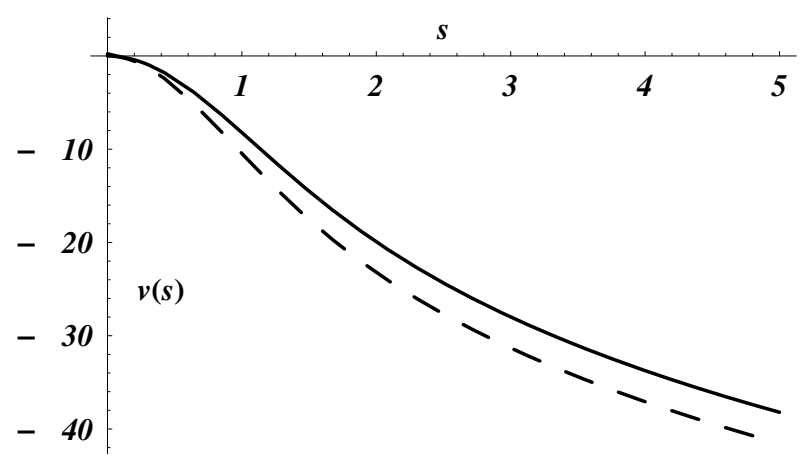

FIG. 4: The space charge potentials $v(s)$ (63) and (52) respectively for a Student (solid line) transverse distribution $\Sigma_{2}\left(22,20 \sigma^{2}\right)$, and for a Gauss (dashed line) distribution. The dimensionless perveance here is $\xi=20$.

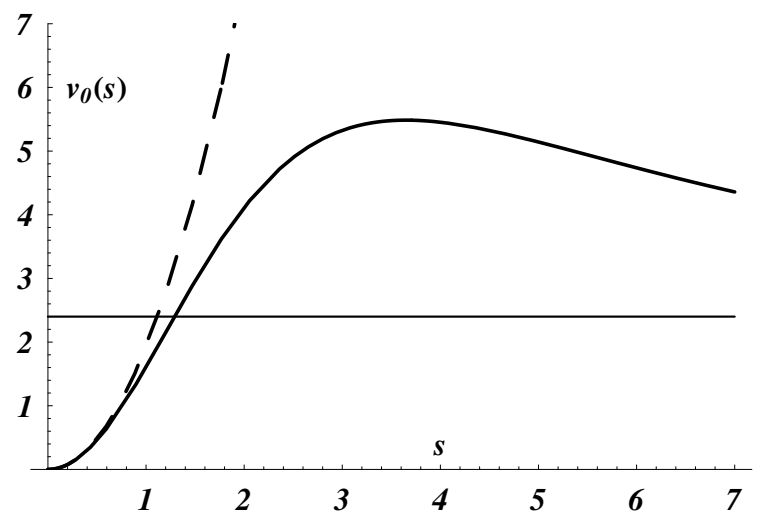

FIG. 5: The control potential $v_{0}(s)$ 64) of a Student $\Sigma_{2}\left(22,20 \sigma^{2}\right)$ (solid line; see FIG. 3) is here compared with that of a Gauss distribution (dashed line) which shows the same behavior near the beam axis.

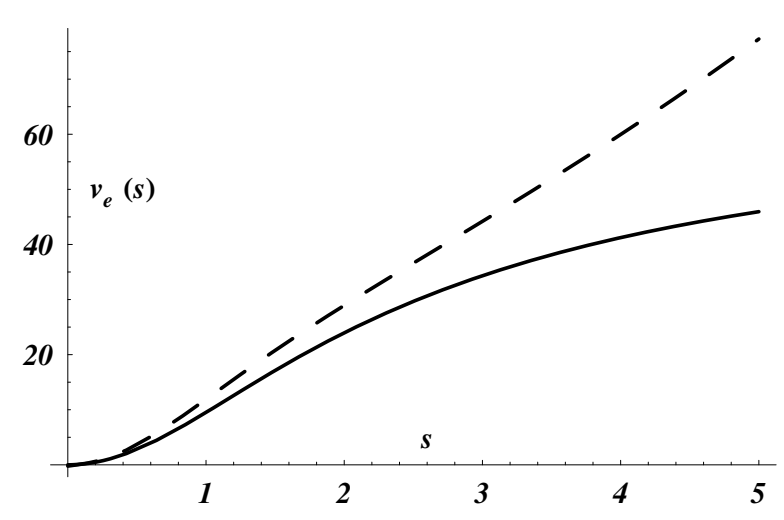

FIG. 6: The total external potential $v_{e}(s)$ that should be applied to get a stationary Student transverse distribution $\Sigma_{2}\left(22,20 \sigma^{2}\right)$ (solid line), compared with that (54) needed for a Gauss distribution (dashed line). transverse direction, but we should remember that in SM we have neither a distribution in the phase space, nor an operator formalism. The momentum and its distribution should then be recovered from the velocity fields (4) and (5) where - since we are dealing with stationary states with $\mathbf{v}=0$ - only the osmotic part is non zero so that

$$
\mathbf{p}=\alpha \frac{\nabla \rho}{\rho}=2 \alpha \frac{\nabla u(r)}{u(r)} .
$$

By supposing now to choose the $\nu$ of our Student laws so that the following integrals exist, we then have

$$
\begin{aligned}
\langle x\rangle & =\int_{0}^{L} \frac{d z}{L} \int_{0}^{2 \pi} \frac{\cos \varphi}{2 \pi} d \varphi \int_{0}^{+\infty} r^{2} u^{2}(r) d r=0 \\
\left\langle x^{2}\right\rangle & =\int_{0}^{L} \frac{d z}{L} \int_{0}^{2 \pi} \frac{\cos ^{2} \varphi}{2 \pi} d \varphi \int_{0}^{+\infty} r^{3} u^{2}(r) d r \\
& =\frac{1}{2} \int_{0}^{+\infty} r^{3} u^{2}(r) d r \\
\left\langle p_{x}\right\rangle & =2 \alpha \int_{0}^{L} \frac{d z}{L} \int_{0}^{2 \pi} \frac{\cos \varphi}{2 \pi} d \varphi \int_{0}^{+\infty} r u(r) u^{\prime}(r) d r=0 \\
\left\langle p_{x}^{2}\right\rangle & =4 \alpha^{2} \int_{0}^{L} \frac{d z}{L} \int_{0}^{2 \pi} \frac{\cos ^{2} \varphi}{2 \pi} d \varphi \int_{0}^{+\infty} r u^{\prime 2}(r) d r \\
& =2 \alpha^{2} \int_{0}^{+\infty} r u^{2}(r) d r
\end{aligned}
$$

so that the standard deviations (uncertainties) are

$$
\begin{aligned}
\Delta x & =\sqrt{\left\langle x^{2}\right\rangle}=\sqrt{\frac{1}{2} \int_{0}^{+\infty} r^{3} u^{2}(r) d r} \\
\Delta p_{x} & =\sqrt{\left\langle p_{x}^{2}\right\rangle}=\sqrt{2 \alpha^{2} \int_{0}^{+\infty} r u^{\prime 2}(r) d r}
\end{aligned}
$$

and the position-momentum covariance is

$$
\begin{aligned}
C & =\left\langle x p_{x}\right\rangle-\langle x\rangle\left\langle p_{x}\right\rangle=\left\langle x p_{x}\right\rangle \\
& =2 \alpha \int_{0}^{L} \frac{d z}{L} \int_{0}^{2 \pi} \frac{\cos ^{2} \varphi}{2 \pi} d \varphi \int_{0}^{+\infty} r^{2} u^{\prime}(r) u(r) d r \\
& =\alpha \int_{0}^{+\infty} r^{2} u^{\prime}(r) u(r) d r
\end{aligned}
$$

In a previous paper [1] we adopted the uncertainty product $\Delta x \cdot \Delta p_{x}$ as a measure of the r.m.s. emittance. As an example let us suppose again that our wave function has the form $u_{0}(r)$ for the harmonic oscillator without space charge given in (45). We then have

$$
\Delta x \cdot \Delta p_{x}=-C=\alpha .
$$

This allows two remarks: first, $\alpha$ plays also the role of a measure of the emittance and hence - as suggested in a previous paper 21] - its value must be linked to the number of particles in the beam; second, the positionmomentum correlation coefficient of a Gaussian beam is

$$
\frac{C}{\Delta x \cdot \Delta p_{x}}=-1
$$


as it was predictable, since in SM the relation between position and momentum for the wave function (45) is linear and negative.

In other models the transverse r.m.s. emittance is calculated by means of the quantity $\sqrt{\Delta x^{2} \Delta p_{x}^{2}-C^{2}}$. In the $\mathrm{KV}$ distribution, since momentum and position are uncorrelated and $\langle x\rangle=\left\langle p_{x}\right\rangle=0$, this estimate becomes $\sqrt{\left\langle x^{2}\right\rangle\left\langle p_{x}^{2}\right\rangle-\left\langle x p_{x}\right\rangle^{2}}$. In the SM model, on the contrary, this is not a good choice: in fact we have shown, at least in our simple example, that $x$ and $p_{x}$ are far to be uncorrelated, and that as a consequence of (70) $\sqrt{\Delta x^{2} \Delta p_{x}^{2}-C^{2}}$ becomes exactly zero. Apparently it is not realistic to take this value as a good estimate of the emittance. On the other hand, for the same gaussian example, the value of the uncertainty product $\Delta x \Delta p_{x}$ is just $\alpha$ which we assume to be a good candidate for the value of the emittance. On the other hand it is easy to calculate the same uncertainty product for a Student distribution $\Sigma_{2}\left(\nu,(\nu-2) \sigma^{2}\right)$ with dimensionless radial distribution (61) and variance $\sigma^{2}$ : in fact a straight application of (68) and (69) brings to the following result

$$
\Delta x \cdot \Delta p_{x}=\alpha \sqrt{\frac{\nu(\nu+2)}{(\nu-2)(\nu+4)}}
$$

Of course, as it is already clear, this value converges to the Gaussian case for large $\nu$, while becomes larger and larger for small $\nu$ values when the shape of the distribution moves away from the Gaussian case.

\section{Weighing the tails}

We can finally compare the length of the tails of Gauss and Student distribution in order do assess the possible halo formation in the second case. Let us consider the probability

$$
P(c)=\int_{c \sigma}^{+\infty} r u^{2}(r) \mathrm{d} r
$$

of being beyond a distance $c \sigma$ ( $\sigma^{2}$ being the variance) away from the beam axis, and calculate this quantity in our two cases. From the Gaussian distribution we have from (45) that

$$
P(c)=e^{-c^{2} / 2}
$$

while in the Student case from (59) we get

$$
P(c)=\left(1+\frac{c^{2}}{\nu-2}\right)^{-\nu / 2}
$$

Now for $c=10$ the Gaussian value is about $1.9 \times 10^{-22}$, while with $\nu=10$ the Student value is about $2.2 \times 10^{-6}$, and with $\nu=22$ the value is $2.8 \times 10^{-9}$. This means that for $\mathcal{N}=10^{11}$ particle per meter of beam, we find practically no particle beyond $10 \sigma$ in the Gaussian case, but about $10^{3}$ particle per meter for a $\nu=22$ Student distribution, and as much as $10^{5}$ for a $\nu=10$ value. It is worthwhile to remember at this point that we got about the same number of particles gone astray in our selfconsistent numerical solutions for a dimensionless perveance of about $\xi=20$ in one of our previous paper [1].

\section{LÉVY-STUDENT PROCESSES}

In our context the Student laws $\Sigma\left(\nu, a^{2}\right)$ are important not only because they promise to better describe the halo by means of their longer tails with respect to usual Gaussian distributions; in fact they constitute an important family of Lévy infinitely divisible (i.d.) laws. At present there is a lot of interest about non-Gaussian Lévy laws in several fields of research (see for example [10, 18] and references quoted therein), but this interest is mostly confined to the stable laws which are in fact an important sub-family of the i.d. laws. The fundamental character of the i.d. laws can be better understood from two different, but strictly correlated standpoints: on the one hand the i.d. laws constitute the more general form of possible limit laws for the generalized Central Limit Theorem; on the other they constitute the class of all the laws of the increments for every stationary, stochastically continuous, independent increments process (Lévy process). These important results (which are briefly discussed in Appendix $\mathrm{A}$ and Appendix B have been achieved by $\mathrm{P}$. Lévy, A.Ya. Khintchin, A. Kolmogorov and other mathematicians from the mid 30's to the mid 40's of the XXth century, but their relevance for the applications has been recognized only in more recent years. One of the characteristics of a non-Gaussian Lévy process is to have trajectories with moving discontinuities (think to the trajectories of a typical Poisson process contrasted with those of a Gaussian Wiener process), and we propose here to describe the trajectories of the particle beam by means of a Lévy-Student process whose discontinuities can possibly account for the relatively rare escape of particles from the beam core. For the sake of simplicity we will limit ourselves in the following to the case of 1-DIM systems representing one single transverse coordinate of our particle beam.

\section{A. The Student i.d. laws}

The ch.f.'s of the laws $\Sigma\left(\nu, a^{2}\right)$, namely the Fourier transform of the densities (55), are

$$
\varphi(\kappa)=2 \frac{|a \kappa|^{\frac{\nu}{2}} K_{\frac{\nu}{2}}(|a \kappa|)}{2^{\frac{\nu}{2}} \Gamma\left(\frac{\nu}{2}\right)}
$$

where $\mathrm{K}_{\alpha}(z)$ is a modified Bessel function. The typical form of these ch.f's (contrasted with the Gauss ch.f.) is shown in FIG. 7 Remark that, since $x$ is a length, the 


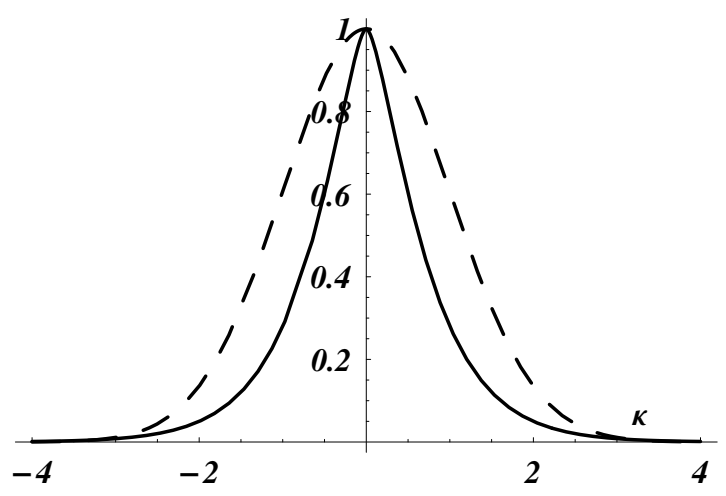

FIG. 7: Typical ch.f. of a Student law $\Sigma(2,2)$ (solid line) compared with the ch.f. of a standard Gauss law $\mathcal{N}(0,1)$ (dashed line).

ch.f. variable $\kappa$ has the dimensions of wave number (inverse of a length). These laws are i.d. but in general are not stable, the unique stable laws among them being the Cauchy laws $\mathcal{C}(a)=\Sigma\left(1, a^{2}\right)$. For $\nu>2$ the Student distributions belong to the domain of attraction of the Gauss law since they have a finite variance. For $\nu \leq 2$ the variance diverges, but it is possible to prove that this notwithstanding they still belong to the Gaussian domain also for $\nu=2$ : albeit this derives from a well known general result 28] a simple proof for our particular case will be given in a subsequent paper. On the other hand for $\nu<2$ the Student laws are attracted by non-Gaussian stable laws characterized by the same value of the parameter $\nu$.

The fact that the Student laws are i.d. - which in itself is not at all a trivial result proved in steps only in the 70's and 80's 29] - shows two kinds of advantages with respect to more common stable laws:

- no stable, non-Gaussian law can have a finite variance, while all Student laws with $\nu>2$ do have a finite variance; this is important since it is not realistic to suppose that empirical distributions (in particular for the particle beams) have infinite variances, but that notwithstanding we will not be obliged to resort to handmade modification (for instance truncated Lévy distributions) as in the case of stable distributions [10];

- the asymptotic behavior of stable, non-Gaussian laws is proportional to $|x|^{-\alpha-1}$ with $\alpha<2$ [18], while the asymptotic behavior of the Student laws is $|x|^{-\nu-1}$ with $\nu>0$; this allows the Student laws - but not the stable laws - to continuously go throughout all the gamut of decay speeds to approximate in a fine tuning the Gaussian behavior as well as we want.

The principal drawback for not being stable is in the subsequent definition of the Lévy-Student process. In fact the ch.f. of the process $\varphi(\kappa, t)$ coincides with (75) only for $t-s=T$, while for $t-s \neq T$ it is no more the ch.f. of a $\Sigma\left(\nu, a^{2}\right)$ law. Hence we explicitly know the form of the increment law only at the time scale $T$ : we know the ch.f. - namely everything we theoretically need - at every time, but we do not have the explicit inverse Fourier transform, and we also know that the laws are no more in the family $\Sigma\left(\nu, a^{2}\right)$. This problem is tempered by the remark that the situation is not better for general stable laws: even in this case, in fact, we do not know the explicit forms of the increment laws not even for one time scale (they are known only in a few precious instances). The unique advantage in the stable case being the fact that all along the time evolution the increment laws remain of the same type, which is not the case for i.d. non stable laws.

\section{B. The Lévy-Student process}

A Lévy process defined by the ch.f. (75) will be called in the following a Lévy-Student process. Taking into account (B3) and (75) we can now state that the transition pdf of a Lévy-Student process is

$$
\begin{aligned}
& p(x, t \mid y, s)= \\
& \frac{1}{2 \pi} \int_{-\infty}^{+\infty} e^{i \kappa(x-y)}\left[2 \frac{|a \kappa|^{\frac{\nu}{2}} K_{\frac{\nu}{2}}(|a \kappa|)}{2^{\frac{\nu}{2}} \Gamma\left(\frac{\nu}{2}\right)}\right]^{\frac{t-s}{T}} \mathrm{~d} \kappa
\end{aligned}
$$

where the improper integral is always convergent since the asymptotic behavior of the ch.f. is

$$
\varphi(\kappa)=\sqrt{2 \pi} \frac{|a \kappa|^{\frac{\nu-1}{2}} e^{-|a \kappa|}\left[1+\mathrm{O}\left(|\kappa|^{-1}\right)\right]}{2^{\frac{\nu}{2}} \Gamma\left(\frac{\nu}{2}\right)}, \quad|\kappa| \rightarrow+\infty
$$

In principle (76) should be enough to calculate everything of our process, but in practice this is an integral that must be treated numerically, but for a few particular cases that will be discussed in a subsequent paper. On the other hand even to produce simulation for the trajectories of our process we should have some simple expression for the transition pdf. At least for this last task, however, we can exploit the fact that when $t-s=T$ the expression (76) can be exactly calculated and coincides with the pdf (55) of a Student $\Sigma\left(\nu, a^{2}\right)$ (remark that even this is not possible for the typical non-Gaussian stable process). This means that we can produce sample trajectories by taking $T$ as the fundamental step of our numerical simulation. In other words we will simulate the sample paths of a process whose increments are exactly Student distributed when observed at the (otherwise arbitrary) time scale $T$. To give a look to these trajectories we produced a simplified model which simulates the solutions of the following two SDE's

$$
\begin{aligned}
\mathrm{d} X(t) & =v(X(t), t) \mathrm{d} t+\mathrm{d} W(t) \\
\mathrm{d} Y(t) & =v(Y(t), t) \mathrm{d} t+\mathrm{d} S(t)
\end{aligned}
$$

where $W(t)$ is a Wiener process, while $S(t)$ is a LévyStudent process. We also fixed the velocity field $v(x, t)$ 


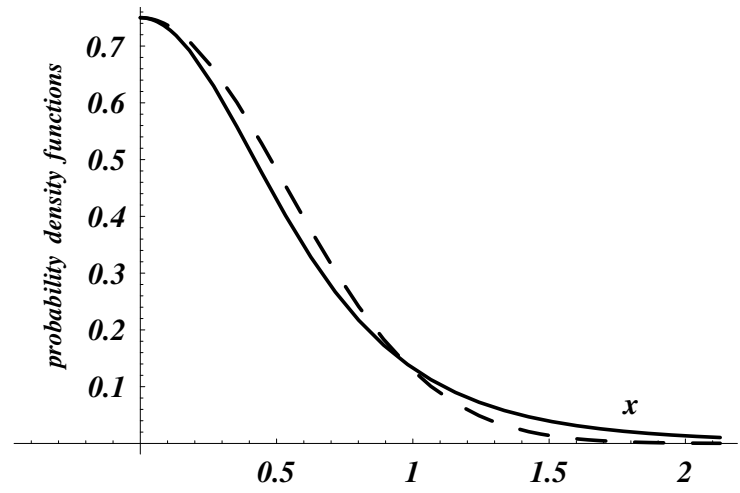

FIG. 8: The pdf's of the increments for the Gaussian processes (SDE (77), dashed line; $\sigma \simeq 0.53$ ) and for the LévyStudent process with law $\Sigma(4,1)$ (SDE (78), solid line; $\sigma \simeq$ $0.71)$. The parameters are chosen so that the two pdf's have the same modal values and similar shapes.

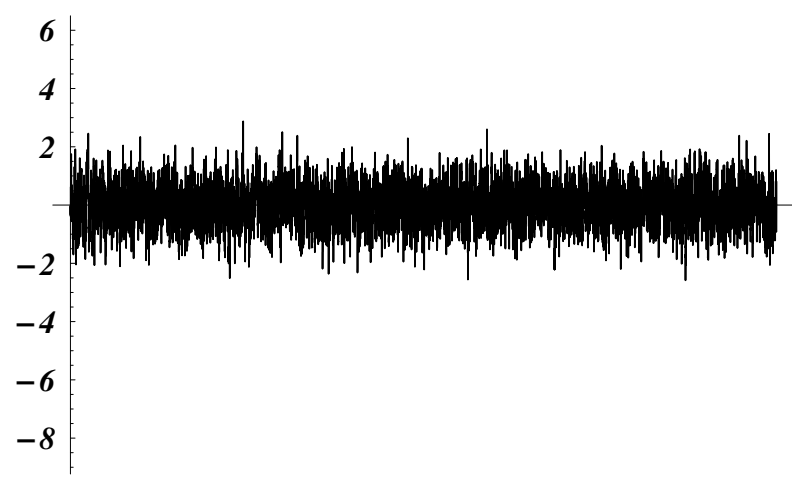

FIG. 9: Typical trajectory of a stationary, Gaussian (Ornstein-Uhlenbeck) process $X(t)$ (see SDE (77)). To compare it with the Student trajectory, the vertical scale has been set equal to that of FIG. 10]

in a suitable way: it will not depend on time $t$, and its value is (for given $b>0$ and $q>0$ )

$$
v(x)=-b x H(q-|x|)
$$

where $H$ is the Heaviside function. This flux will attract the trajectory toward the origin when $|x| \leq q$, and will allow the movement to be completely free for $|x|>q$. The forms of the typical pdf's used in our simulations are shown in FIG. 8 In a simplified model for a collimated beam this will then produce a stationary, Ornstein-Uhlenbeck process for the SDE (77) if the intensity of the Gaussian noise is not too large. The process solution of the SDE (78) will instead have different characteristics. Let us suppose to fix the ideas that the two parameters defining the velocity field are $b=0.35$ and $q=10$. The FIG. 9] displays a typical trajectory of a $10^{4}$ steps solution $X(t)$ of (77) when the variance of the Gaussian distributed increments is $\sigma^{2}=0.28$. In

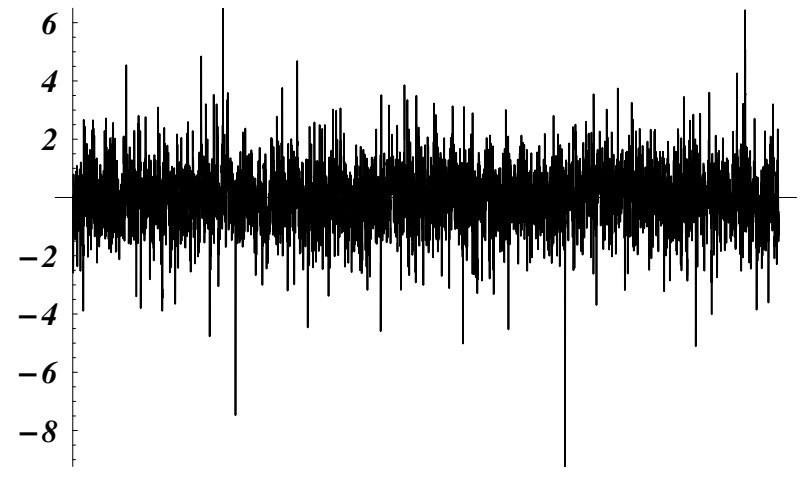

FIG. 10: Typical trajectory of a stationary, Student process $Y(t)$ (see $\operatorname{EDS}$ (78), $\nu=4$ and $a=1$ ).

our simplified 1-DIM model of the transverse dynamics of a particle beam this means that the trajectories always stay inside the beam core. Let us then take as law for the increments of (78) a Student distribution $\Sigma(4,1)$ : its pdf looks not very different from that of the previous Gaussian distribution, as the FIG. 8 clearly show. That notwithstanding the process $Y(t)$ differs in several respects from $X(t)$. Indeed not only the typical trajectory displayed in FIG. 10] shows a wider dispersion of its values and a few larger spikes. The principal difference is rather in the fact that while the trajectories of $X(t)$ show a remarkable stability in their statistical behavior, the paths of $Y(t)$ have the propensity to make occasional excursions far away from the beam core (see FIG. 11), and seldom they also definitely drift away from the core (see FIG. 12). This depends of course on the mentioned properties of the trajectories of a non-Gaussian Lévy process, and in particular on the fact that they are only stochastically, and not pathwise continuous, namely that they contain occasional jumps. The frequency and the size of these jumps can also be fine tuned by suitably choosing the values of the parameters of the law $\Sigma\left(\nu, a^{2}\right)$ of the increments. It is this feature of a Lévy-Student process that suggests to adopt this model to describe the rare escape of particles away from the beam core.

\section{CONCLUSIONS}

In the previous sections we have introduced the Student laws in our SM model for the particle beam dynamics first of all in order to make use of their features depending on their enhanced variance. In particular we have shown that the longer tails with respect to the similar Gaussian distributions can help to account for the finding of a larger than expected number of particles removed far away from the beam core.

It should be remarked, however, that all along the Section IV our processes were Gaussian processes since the underlying SDE (10) is still powered by a Brownian noise. 


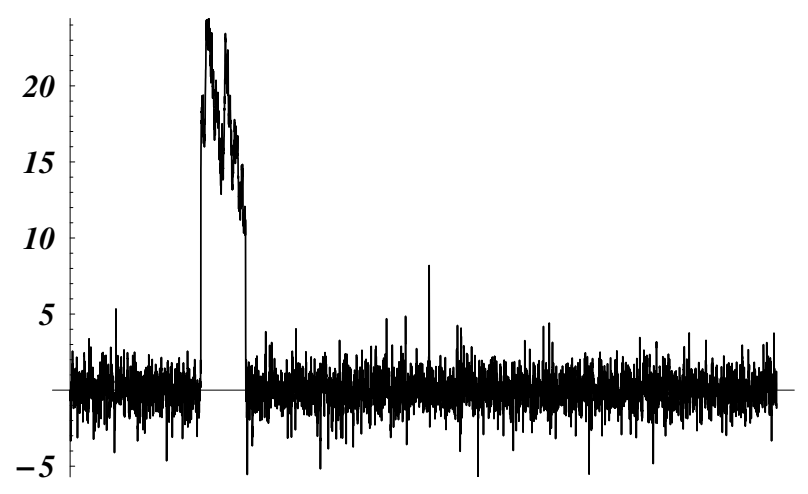

FIG. 11: Occasional trajectory of a stationary, Student process $Y(t)$ (see EDS (78), $\nu=4$ ) with a temporary excursion out of the core.

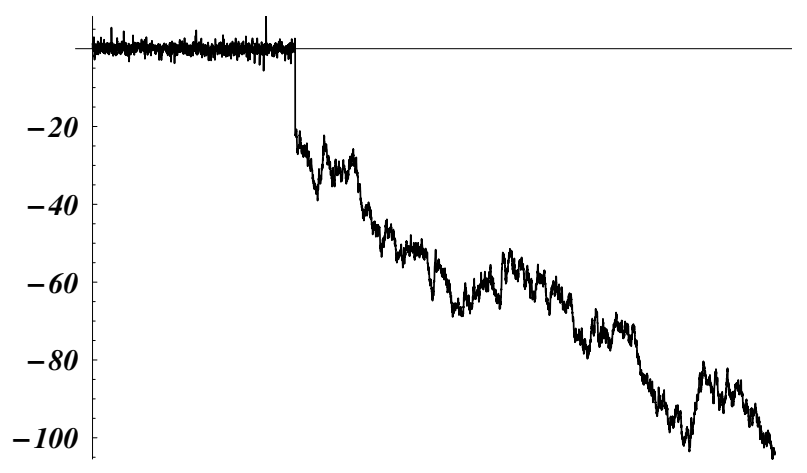

FIG. 12: Rare, but possible trajectory of a stationary, Student process $Y(t)$ (see EDS (78), $\nu=4$ ): here the particle definitely drifts away from the core.

This is true even in the Section IVB where we first introduced the Student laws (55) as stationary distributions of the process. It is only in the Section $\nabla$ that we introduced a new kind of SDE with a Lévy-Student noise. The more relevant feature of these processes is the fact that their trajectories make jumps: indeed this can become a model for the halo formation in the beams. From a physical point of view these jumps can be produced by occasional hard collisions among the beam particles, the probability of these collisions growing with the intensity of the beam. In some sense it is not only the variance of the transverse distribution of the beam which principally rules the emergence of a halo: in the simulations produced here the variances of the Gaussian and of the Student processes were roughly the same. Rather it is the qualitative character of the process which accounts for the rare escape of the particles from the beam core. For a process produced by a Gaussian noise (a process pathwise continuous: almost every trajectory is everywhere continuous) there is no chance to observe trajectories going out of a well collimated beam. On the contrary, for a process produced by a Lévy-Student noise (a process only stochastically continuous: trajectories can have jumps) occasionally the jump is large enough to put the particle out of the stream. Of course the frequency and the size of these jumps depend on the parameters $\nu$ and $a$ of the process: the jumps tend to be smaller and less frequent when the $\Sigma\left(\nu, a^{2}\right)$ distributions approximate a Gaussian law. In our opinion it would be very interesting to explore the possibility that the processes underlying the intense beam dynamics be ruled by some sort of Lévy-Student noise rather than by the usual Gaussian noise. It is then important to point out that a few numerical evidences [20] begin to emerge which confirm this conjecture.

These remarks point to several research directions. First of all it is important to better study the LévyStudent process in itself: for example a knowledge of the Lévy-Khintchin functions of the Student laws would be relevant to the fine tuning of the frequency and the size of the trajectory jumps. On the other hand even the differential form of its Chapman-Kolmogorov equation 30. would be instrumental to discuss the time evolution of the process. Then it must be remarked that at present we have just defined the Lévy-Student process, but we added no dynamics: it is as if we have the Wiener process, but no Stochastic Mechanics or any other dynamical model added to this kinematics. In other words we need to build a new generalized SM for the Lévy-Student processes. Finally it would be important at this point to have empirical or numerical data able to corroborate the hypothesis that the increments of the transverse variables of a beam are in fact distributed according to a Student law, rather than according to the usual Gaussian law.

\section{Acknowledgments}

We would like to thank Dr. C. Benedetti, Prof. F. Mainardi, Prof. G. Turchetti and Dr. A. Vivoli for useful comments and suggestions.

\section{APPENDIX A: INFINITELY DIVISIBLE AND STABLE LAWS}

The relevant mathematical concepts used in this paper are better discussed in the framework of the theory of the addition of independent random variables (r.v.): for more details see [31, 32, 33]. In the following we will describe the law $\mathcal{L}$ of a r.v. $X$ by giving her characteristic function (ch.f.)

$$
\varphi(\kappa)=\mathbf{E}\left(e^{i \kappa X}\right)
$$

where $\mathbf{E}(\cdot)$ is the expectation under the law $\mathcal{L}$. When $\mathcal{L}$ has a pdf $f(x)$, then $\varphi(\kappa)$ is just its Fourier transform. It is well known that the law $\mathcal{L}$ of the sum of $n$ independent r.v.'s with laws $\mathcal{L}_{1}, \ldots, \mathcal{L}_{n}$ has a ch.f. which is the product 
of the ch.f.'s of the component laws:

$$
\varphi(\kappa)=\varphi_{1}(\kappa) \cdot \ldots \cdot \varphi_{n}(\kappa)
$$

On the other hand we say that a law $\mathcal{L}$ is decomposed in the laws $\mathcal{L}_{1}, \ldots, \mathcal{L}_{n}$ when its ch.f. can be written as a product A1 of the ch.f.'s of its components. This already allows us to introduce two fundamental concepts: a law $\mathcal{L}$ with ch.f. $\varphi$ is said to be i.d. when for every $n$ there is a law $\mathcal{L}_{n}$ with ch.f. $\varphi_{n}$ such that $\varphi=\varphi_{n}^{n}$. In other words this means that for every $n$ a r.v. $X$ with law $\mathcal{L}$ can always be decomposed in the sum of $n$ independent r.v.'s all with the same law $\mathcal{L}_{n}$ (identically distributed). Remark, however, that in general the laws $\mathcal{L}_{n}$ are not of the same type as $\mathcal{L}$. Let us remember here that we say that two laws are of the same type when we get one from the other by means of a centering and a rescaling; in other words, if $\varphi(\kappa)$ in a ch.f., then all the ch.f.'s of the same type have the form $e^{i a \kappa} \varphi(b \kappa)$ for every $a$ and $b>0$. For instance all the Gaussian laws $\mathcal{N}\left(\mu, \sigma^{2}\right)$ belong to the same (Gaussian) type; on the contrary the Poisson laws $\mathcal{P}(\lambda)$ with different values of $\lambda$ do not belong to the same type. Now, a law $\mathcal{L}$ is said to be stable when it is i.d. and the component laws are of the same type as $\mathcal{L}$. More precisely a ch.f. $\varphi(\kappa)$ is stable when for every $b, b^{\prime}>0$ there exist $a$ and $c$ such that

$$
\varphi(c \kappa)=e^{i a \kappa} \varphi(b \kappa) \varphi\left(b^{\prime} \kappa\right) .
$$

As an example: the Gaussian and the Cauchy laws are stable; the Poisson laws are instead only i.d. The families of i.d. and stable laws are completely characterized: in fact the celebrated Lévy-Khintchin formula gives the more general form for the ch.f.'s of these two classes; however, while in the case of the stable laws these ch.f.'s (albeit not in general the laws themselves) are explicitly known in terms of elementary functions, for the i.d. laws the ch.f.'s are given through an integral containing a function $L(x)$ (Lévy function) associated to every particular law. But for a few classical cases the Lévy functions of the i.d. laws are not known.

\section{APPENDIX B: CENTRAL LIMIT THEOREM AND LÉVY PROCESSES}

Let us consider the sequence of r.v.'s $X_{n, k}$ with $n \in \mathbb{N}$ and $k=1, \ldots, n$ with $X_{n, 1}, \ldots, X_{n, n}$ independent for every $n$. The modern formulation of the Central Limit Problem asks to find the more general laws which are limits of the laws of the consecutive sums

$$
S_{n}=\sum_{k=1}^{n} X_{n, k}
$$

Remark that these sums generalize the usual partial sums of the classical Central Limit Theorem in that: when we go from $S_{n}$ to, say, $S_{n+1}$, the first $n$ terms do not in general remain the same: for example $X_{n .1}$ does not coincide with $X_{n+1.1}$. Under very general technical conditions the Central Limit Theorem now states that the family of all the limit laws of the consecutive sums (B1) coincides with the family of i.d. laws. The stable laws come into play only when we specialize the form of our consecutive sums: when we have

$$
X_{n, k}=\frac{X_{k}}{a_{n}}-\frac{b_{n}}{n}
$$

where $a_{n}$ and $b_{n}$ are sequences of numbers, and $X_{k}$ are independent r.v.'s, the consecutive sums take the form of the usual normed sums (centered and rescaled sums of independent r.v.'s)

$$
S_{n}=\frac{S_{n}^{*}}{a_{n}}-b_{n}, \quad S_{n}^{*}=\sum_{k=1}^{n} X_{k} .
$$

Then, if the $X_{k}$ are also identically distributed, the family of the limit laws of the normed sums (B2) coincides with the family of the stable laws. The classical (Gaussian) Central Limit Theorem is an example of convergence toward a stable law; on the other hand the Poisson Theorem (convergence of Binomial laws toward Poisson laws) is an example of convergence toward an i.d. law. Every stable law has its own domain of attraction, namely the set of laws attracted by it in the sense of the convergence of normed sums (B2) of independent r.v.'s all distributed as the attracted law. It can be proved that all the laws with finite variance are in the domain of attraction of the Gauss law, and that a law can be attracted by a nonGaussian stable law only if it has infinite variance.

The general formulation of the Central Limit Theorem is strictly connected to the definition of the processes with independent increments (decomposable processes). It is apparent in fact that if the increments $\Delta X(t)=X(t+\Delta t)-X(t)$ for non superposed intervals are independent, the previous forms of the Central Limit Theorem imply that the laws of the increments must be i.d. laws. Moreover, since the decomposable process are also Markov processes, the laws of the increments are also all that is needed to completely define them. If a decomposable processes $X(t)$ is stationary (namely the law of $X(t+s)-X(s)$ does not depend on $s$ ) and stochastically continuous (namely for every $t$ we have $X(t+\Delta t)-X(t) \rightarrow 0$ in probability when $\Delta t \rightarrow 0$ ) we will call it a Lévy process. Remark that a Poisson process is a Lévy process since, despite its discontinuities, it is stochastically continuous. In fact these discontinuities do not impair the stochastic continuity of the process because they are moving (as opposed to fixed) discontinuities. On the other hand it is possible to prove that only the Gaussian Lévy processes (for example the Wiener, or the Ornstein-Uhlenbeck processes) are pathwise continuous, namely: almost every sample path is everywhere continuous (there are not even moving discontinuities). Now, if $\varphi(\kappa)$ is the ch.f. of an i.d. law and $T$ is a suitable time constant, it is possible to prove that $[\varphi(\kappa)]^{\Delta t / T}$ is the ch.f. of the increments $\Delta X(t)$ of a Lévy process. 
Hence, if the process has a pdf, the stationary transition pdf is

$$
p(x, t \mid y, s)=\frac{1}{2 \pi} \mathrm{PV} \int_{-\infty}^{+\infty} e^{i \kappa(x-y)}[\varphi(\kappa)]^{\frac{t-s}{T}} \mathrm{~d} \kappa
$$

so that, at least in principle, we know all that is needed to define the process.

The sample paths of a Lévy process are also well characterized: it is possible in fact to prove that almost all trajectories are bounded and are continuous with the exception of a countable set of moving jumps (first kind discontinuities). Then, let us suppose that $L_{t}(x)$ is the Lévy-Khintchin function of the i.d. law of the increment $X(s+t)-X(s)$ : if $\nu_{t}(x)$ is the random number of the jumps in $[s, s+t)$ of height in absolute value larger than $x>0$, it is possible to prove that

$$
\left|L_{t}(x)\right|=\mathbf{E}\left(\nu_{t}(x)\right)
$$

so that the Lévy-Khintchin function of an i.d. law plays also the role of a measure of the frequency and height of the trajectory jumps.
[1] N. Cufaro Petroni, S. De Martino, S. De Siena, and F. Illuminati, Phys. Rev. ST Accel. Beams 6, 034206 (2003); N. Cufaro Petroni, S. De Martino, S. De Siena and F. Illuminati, in Quantum aspects of beam physics 2003, P. Chen et al. eds. (World Scientific, Singapore, 2004) p. 36.

[2] N. Cufaro Petroni, S. De Martino, S. De Siena and F. Illuminati, in Proceedings of the European particle accelerator conference - EPAC04 (EPS-AG/CERN 2004), p. 2056.

[3] H. Koziol, Los Alamos M. P. Division Report No. MP-375-1 (1975).

[4] M. Reiser, C. Chang, D. Kehne, K. Low, T. Shea, H. Rudd, and J.Haber, Phys. Rev. Lett. 61, 2933 (1988).

[5] R.L. Gluckstern, Phys. Rev. Lett. 73, 1247 (1994); R.L. Gluckstern, W.-H. Cheng and H.Ye, Phys. Rev. Lett. 75, 2835 (1995); R.L. Gluckstern, W.-H. Cheng, S.S. Kurennoy, and H.Ye, Phys. Rev. E 54, 6788 (1996); H. Okamoto and M. Ikegami, Phys. Rev. E 55, 4694 (1997); R.L. Gluckstern, A.V. Fedotov, S.S. Kurennoy, and R. Ryne, Phys. Rev. E 58, 4977 (1998); T.P. Wangler, K.R. Crandall, R. Ryne, and T.S. Wang, Phys. Rev. ST-AB 1, 084201 (1998); A.V. Fedotov, R.L. Gluckstern, S.S. Kurennoy, and R.Ryne, Phys. Rev. ST-AB 2, 014201 (1999); M. Ikegami, S. Machida, and T. Uesugi, Phys. Rev. ST-AB 2, 124201 (1999); Quiang and R. Ryne, Phys. Rev. ST-AB 3, 064201 (2000).

[6] O. Boine-Frankenheim and I. Hofmann Phys. Rev. STAB 3, 104202 (2000); L. Bongini, A. Bazzani, G. Turchetti, and I. Hofmann Phys. Rev. ST-AB 4, 114201 (2001); A.V. Fedotov and I. Hofmann Phys. Rev. ST-AB 5, 024202 (2002).

[7] T. Wangler, RF linear accelerators, (J. Wiley, New York, 1998)

[8] L.D. Landau and E.M. Lifchitz, Cintique physique, (MIR, Moscow, 1990).

[9] F. Ruggiero, Ann. Phys. (N.Y.) 153, 122 (1984); F. Ruggiero, E. Picasso and L.A. Radicati, Ann. Phys. (N. Y.) 197, 396 (1990); J. Struckmeier, Phys. Rev. ST-AB 3, 034202 (2000).

[10] W. Paul and J. Baschnagel, Stochastic Processes: From Physics to Finance, (Springer, Berlin, 2000)

[11] E. Nelson, Dynamical theories of Brownian motion (Princeton University Press, Princeton N. J., 1967); E. Nelson, Quantum Fluctuations (Princeton University Press, Princeton N. J., 1985).

[12] F. Guerra, Phys. Rep. 77, 263 (1981).

[13] F. Guerra and L. M. Morato, Phys. Rev. D 27, 1774
(1983).

[14] S. Albeverio, Ph. Blanchard and R. Høgh-Krohn, Expo. Math. 4, 365 (1983).

[15] R. Fedele, G. Miele and L. Palumbo, Phys. Lett. A 194, 113 (1994), and references therein; S.I. Tzenov, Phys. Lett. A 232, 260 (1997).

[16] S.A. Khan and M. Pusterla, Eur. Phys. J. A 7, 583 (2000).

[17] L. Morato, J. Math. Phys. 23 (1982) 1020.

[18] R.N. Mantegna and H.E. Stanley, An Introduction to Econophysics (Cambridge U.P. 2000)

[19] C.K. Allen, K.C.D. Chan, P.L. Colestock, K.R. Crandall, R.W. Garnett, J.D. Gilpatrick, W. Lysenko, J. Qiang, J.D. Schneider, M.E. Schulze, R.L. Sheffield, H.V. Smith and T.P. Wangler, Phys. Rev. Lett. 89 (2002) 214802.

[20] A. Vivoli, C. Benedetti and G. Turchetti, Time Series Analysis of Coulomb Collisions in a Beam Dynamics Simulation, Workshop COULOMB'05, September 12-16, 2005, Senigallia.

[21] S. De Martino, S. De Siena, and F. Illuminati, Physica A 271, 324 (1999); N. Cufaro Petroni, S. De Martino, S. De Siena, and F. Illuminati, Phys. Rev. E 63, 016501 (2000); N. Cufaro Petroni, S. De Martino, S. De Siena and F. Illuminati, in Quantum aspects of beam physics 2K, P. Chen ed. (World Scientific, Singapore, 2002) p. 507.

[22] N. Cufaro Petroni, S. De Martino, S. De Siena, and F. Illuminati, Int. J. Mod. Phys. B 18, 607 (2004)

[23] E. Madelung, Z. Physik 40, 332 (1926); D. Bohm, Phys. Rev. 85, 166, 180 (1952).

[24] N. Cufaro Petroni, S. De Martino, S. De Siena, and F. Illuminati, J. Phys. A 32, 7489 (1999); N. Cufaro Petroni, S. De Martino, S. De Siena and F. Illuminati, in Quantum aspects of beam physics 1998, P. Chen ed. (World Scientific, Singapore, 1999) p. 710; N. Cufaro Petroni, S. De Martino, S. De Siena, R. Fedele, F. Illuminati and S. I. Tzenov, in Proceedings of the European particle accelerator conference - EPAC98, S. Myers et al. eds. (IoP Publishing, Bristol, 1998), p. 1259.

[25] N. Cufaro Petroni and F. Guerra, Found. Phys. 25, 297 (1995); N. Cufaro Petroni, in Quantum Communications and Measurement, V.P. Belavkin et al. eds. (Plenum, New York, 1995), p. 43; N. Cufaro Petroni, S. De Martino and S. De Siena, in New Perspectives in the Physics of Mesoscopic Systems, S. De Martino et al. eds. (World Scientific, Singapore 1997) p. 59.

[26] N. Cufaro Petroni, S. De Martino and S. De Siena, Phys. 
Lett. A 245, 1 (1998).

[27] L.D. Landau and E.M. Lifchitz, Mécanique Quantique, (MIR, Moscow, 1988).

[28] J.-P. Bouchaud and A. Georges, Phys. Rep. 195, 127 (1990).

[29] E. Grosswald, Ann. Prob. 4, 680 (1976); E. Grosswald, Z. Wahrsch. 36, 103 (1976); M.E.H. Ismail, Ann. Prob. 5, 582 (1977); L. Bondesson, Ann. Prob. 7, 965 (1979); J. Pitman and M. Yor, in Stochastic Integrals (D. Williams editor), L. N. Math. 851, 285 (Springer, 1981); L. Bondesson, Generalized Gamma Convolutions and Related Classes of Distributions and Densities, L. N. Stat. 76
(Springer, 1992); J. Pitman, Electr. J. Prob. 4 (1999) paper 11.

[30] C.W. Gardiner, Handbook of Stochastic Methods (Springer, 1997).

[31] M. Loève, Probability Theory I and II (Springer, 1977 and 1978).

[32] W. Feller, An Introduction to Probability Theory and Its Applications I and II (J. Wiley \& Sons, 1968 and 1971).

[33] B.V. Gnedenko and A.N. Kolmogorov, Limit distributions for sums of independent random variables (Addison-Wesley, 1968). 